\title{
REACTION-DIFFUSION EQUATIONS ON COMPLEX NETWORKS AND TURING PATTERNS, VIA $p$-ADIC ANALYSIS
}

\author{
W. A. ZÚÑIGA-GALINDO
}

\begin{abstract}
Nakao and Mikhailov proposed using continuous models (mean-field models) to study reaction-diffusion systems on networks and the corresponding Turing patterns. This work aims to show that $p$-adic analysis is the natural tool to carry out this program. This is possible due to the fact that the discrete Laplacian attached to a network turns out to be a particular case of a $p$-adic Laplacian. By embedding the graph attached to the system into the field of $p$-adic numbers, we construct a continuous $p$-adic version of a reactiondiffusion system on a network. The existence and uniqueness of the Cauchy problems for these systems are established. We show that Turing criteria for the $p$-adic continuous reaction-diffusion system remain essentially the same as in the classical case. However, the properties of the emergent patterns are very different. The classical Turing patterns consisting of alternating domains do not occur in the $p$-adic continuous case. Instead of this, several domains (clusters) occur. Multistability, that is, coexistence of a number of different patterns with the same parameter values occurs. Clustering and multistability have been observed in the computer simulations of large reaction-diffusion systems on networks. Such behavior can be naturally explained in the $p$-adic continuous model, in a rigorous mathematical way, but not in the original discrete model.
\end{abstract}

\section{INTRODUCTION}

Pattern-forming, reaction-diffusion systems in continuous media, are typically described by a system of PDEs of the form

$$
\left\{\begin{array}{l}
\frac{\partial u(x, t)}{\partial t}=f(u, v)+\varepsilon \Delta u(x, t) \\
\frac{\partial v(x, t)}{\partial t}=g(u, v)+\varepsilon d \Delta v(x, t),
\end{array}\right.
$$

where $x \in \mathbb{R}^{n}, t \geq 0$, and $u(x, t), v(x, t)$ are local densities of two chemical species, the functions $f$ and $g$ specify the local dynamics of $u$ and $v$, and $\varepsilon, \varepsilon d$ are the corresponding diffusion coefficients. Typically $u$ corresponds to an activator, which autocatalytically enhances its own production, and $v$ an inhibitor that suppresses $u$. The system is initially considered to be at a steady state $\left(u_{0}, v_{0}\right)$ where $f\left(u_{0}, v_{0}\right)=g\left(u_{0}, v_{0}\right)=0$. The Turing instability sets up once the system is subjected to heterogeneous perturbations. If the perturbation is homogeneous, the system will reach again the steady state $\left(u_{0}, v_{0}\right)$. Moreover, one has to take care

2020 Mathematics Subject Classification. Primary: 35R02, 35K57, 47S10. Secondary: 05C82, 92C42.

Key words and phrases. Reaction-diffusion equations, complex networks, Turing patterns, $p$-adic analysis, Markov processes.

The author was partially supported by Conacyt Grant No. 250845 . 
of the difference between a Turing instability and the possibly resulting Turing patterns. Indeed, a Turing instability could give rise to waves or even to another homogeneous equilibrium, [46], [31, Chapter 2]. The existence of Turing patterns requires that the parameter $d$ exceeds a certain threshold $d_{c}$. This event drives to a spontaneous development of a spatial pattern formed by alternating activator-rich and activator-poor patches. Turing instability in activator-inhibitor systems establishes a paradigm of non-equilibrium self-organization, which has been extensively studied for biological and chemical processes.

In the 70s, Othmer and Scriven started the study of the Turing instability in networkorganized systems [36]-[37]. Since then, reaction-diffusion models on networks have been studied intensively, see e.g. [2], [13], [14], [18], [29], [30], [32], [36], 337, [41], [47], [49], [50], and the references therein. In the discrete case, the continuous media is replaced by a network (an unoriented graph $\mathcal{G}$, which plays the role of discrete media) composed by $\# V(\mathcal{G})$ independent nodes (vertices) that interact via diffusive transport on $\# E(\mathcal{G})$ links (edges). The analog of operator $\Delta$ is the Laplacian of the graph $\mathcal{G}$, which is defined as

$$
\left[L_{J I}\right]_{J, I \in V(\mathcal{G})}=\left[A_{J I}-\gamma_{I} \delta_{J I}\right]_{J, I \in V(\mathcal{G})},
$$

where $\left[A_{J I}\right]_{J, I \in V(\mathcal{G})}$ is the adjacency matrix of $\mathcal{G}$ and $\gamma_{I}$ is the degree of $I$. The network analogue of (1.1) is

$$
\left\{\begin{array}{l}
\frac{\partial u_{J}}{\partial t}=f\left(u_{J}, v_{J}\right)+\varepsilon \sum_{I} L_{J I} u_{I} \\
\frac{\partial v_{J}}{\partial t}=g\left(u_{J}, v_{J}\right)+\varepsilon d \sum_{I} L_{J I} v_{I} .
\end{array}\right.
$$

In the last fifty years, Turing patterns produced by reaction-diffusion systems on networks have been studied intensively, mainly by physicists, biologists and engineers, see e.g. [13], [18], [29], [32], [36], [37], [50] and the references therein. Nowadays, there is a large amount of experimental results about the behavior of these systems, obtained mainly via computer simulations using large random networks. The investigations of the Turing patterns for large random networks have revealed that, whereas the Turing criteria remain essentially the same, as in the classical case, the properties of the emergent patterns are very different. In [32], by using a physical argument, Nakao and Mikhailov establish that Turing patterns with alternating domains cannot exist in the network case, and only several domains (clusters) occur. Multistability, that is, coexistence of a number of different patterns with the same parameter values, is typically found and hysteresis phenomena are observed. They used mean-field approximation to understand the Turing patterns when $d>d_{c}$, and proposed that the mean-field approximation is the natural framework to understand the peculiar behavior of the Turing patterns on networks.

The central goal of this work is to show the existence of a new $p$-adic continuous version of the system (1.3) which provides a very good approximation of the original system. This is a consequence of the fact that the discrete Laplacian attached to a network turns out to be a particular case of a $p$-adic Laplacian. We establish from a mathematical perspective, that the program of Nakao and Mikhailov proposed in 32] for understanding the peculiar behavior of 
the Turing patterns on networks using mean-field approximations (i.e. a continuous version of $(1.3)$ ) is possible, but it requires $p$-adic analysis. By 'embedding the graph' $\mathcal{G}$ into $\mathbb{Q}_{p}$, the field of $p$-adic numbers, we construct a family of continuous $p$-adic versions of the system (1.3), which can be studied rigorously by using the classical semigroup theory, see e.g. [28], [38], in this way, we are able to study the original system (1.3) and to obtain a new $p$-adic continuous version of it, which corresponds to a 'mean-field approximation' of the original system (1.3).

From now on $p$ denotes a fixed prime number. A $p$-adic number is a series of the form

$$
x=x_{-k} p^{-k}+x_{-k+1} p^{-k+1}+\ldots+x_{0}+x_{1} p+\ldots, \text { with } x_{-k} \neq 0,
$$

where the $x_{j} \mathrm{~s}$ are $p$-adic digits, i.e. numbers in the set $\{0,1, \ldots, p-1\}$. The set of all possible series of the form (1.4) constitutes the field of $p$-adic numbers $\mathbb{Q}_{p}$. There are natural field operations, sum and multiplication, on series of the form (1.4), see e.g. [23]. There is also a natural norm in $\mathbb{Q}_{p}$ defined as $|x|_{p}=p^{k}$, for a nonzero $p$-adic number of the form (1.4). The field of $p$-adic numbers with the distance induced by $|\cdot|_{p}$ is a complete ultrametric space. The ultrametric property refers to the fact that $|x-y|_{p} \leq \max \left\{|x-z|_{p},|z-y|_{p}\right\}$ for any $x, y, z \in \mathbb{Q}_{p}$. We denote by $\mathbb{Z}_{p}$ the unit ball, which consists of all series with expansions of the form (1.4) with $k \geq 0$.

We identify each vertex of $\mathcal{G}$ with a $p$-adic number of the form

$$
I=I_{0}+I_{1} p+\ldots+I_{N-1} p^{N-1}
$$

where the $I_{j}$ s are $p$-adic digits. The parameter $N$ is fixed along the article. We denote by $G_{N}^{0}$ the set of all $p$-adic integers of the form (1.5) which correspond to the vertices of $\mathcal{G}$. In this way, we construct a $p$-adic parametrization of the adjacency matrix of $\mathcal{G}$. Our constructions depend only on the adjacency matrix of the graph $\mathcal{G}$, for this reason, the tree-like structure of $\mathbb{Q}_{p}$ does not play any role in our construction.

We denote by $\Omega\left(p^{N}|x-I|_{p}\right)$ the characteristic function of the ball centered at $I$ with radius $p^{-N}$, which corresponds to the set $I+p^{N} \mathbb{Z}_{p}$. Now, we attach to $\mathcal{G}$ the open compact subset $\mathcal{K}_{N}$ defined as the disjoint union of the balls $I+p^{N} \mathbb{Z}_{p}$ for $I \in G_{N}^{0}$, and a finite dimensional real vector space $X_{N}$ generated by the functions $\left\{\Omega\left(p^{N}|x-I|_{p}\right)\right\}_{I \in G_{N}^{0}}$. This is the space of continuous functions on $\mathcal{G}$. There exists a kernel $J_{N}(x, y)$, which is a linear combination of functions of type $\Omega\left(p^{N}|x-I|_{p}\right) \Omega\left(p^{N}|y-J|_{p}\right), I, J \in G_{N}^{0}$, such that the operator $\boldsymbol{L}_{N}: X_{N} \rightarrow X_{N}$ is defined as

$$
\boldsymbol{L}_{N} \varphi(x)=\int_{\mathcal{K}_{N}}(\varphi(y)-\varphi(x)) J_{N}(x, y) d y
$$

where $d y$ denotes the normalized Haar measure of the locally compact group $\left(\mathbb{Q}_{p},+\right)$, is represented by the matrix $\left[L_{J I}\right]_{J, I \in G_{N}^{0}}$, see (1.2).

The space $X_{N}$ (endowed with the supremum norm) plays the role of a mesh, which can be refined as much as we want. Given $M>N$, we can subdivide each ball $I+p^{N} \mathbb{Z}_{p}$, with $I \in G_{N}^{0}$, into $p^{M-N}$ disjoint balls $I_{j}+p^{M} \mathbb{Z}_{p}$. In this way we construct new functions of 
type $\sum_{I_{j}} c_{I_{j}} \Omega\left(p^{M}\left|x-I_{j}\right|_{p}\right)$, which form an $\mathbb{R}$-vector space, denoted as $X_{M}$, of dimension $p^{M-N}\left(\# G_{N}^{0},\right)$. We endow $X_{M}$ with the supremum norm. Then $X_{N}$ is continuously embedded, as a Banach space, into $X_{M}$. Furthermore, operator $\boldsymbol{L}_{N}$ has a natural extension $\boldsymbol{L}_{M}$ to $X_{M}$ given by the right-hand side of formula (1.6) .

We set $X_{\infty}$ for the vector space of real-valued, continuous functions on $\mathcal{K}_{N}$, endowed with the supremum norm. Then $X_{M}$ is continuously embedded, as a Banach space, into $X_{\infty}$, and $\cup_{M \geq N} X_{M}$ is dense in $X_{\infty}$. Furthermore, operator $\boldsymbol{L}_{M}$ has an extension $\boldsymbol{L}$ to $X_{\infty}$ given by the right-hand side of formula (1.6), which is a linear bounded operator. In this way on each $X_{\bullet}$, we have an operator $\boldsymbol{L}_{\bullet}$, where the $\operatorname{dot}$ means $N, M$ with $M>N$ or $\infty$, and a continuous version of the system (1.3):

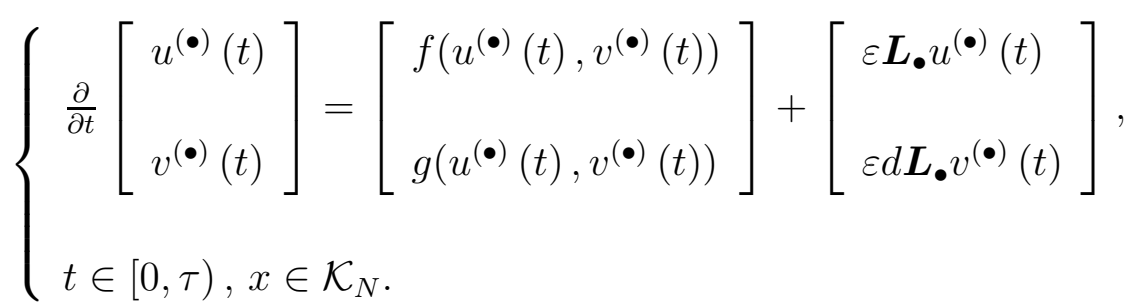

We study the Cauchy problem attached to (1.7), when the initial datum belongs to a sufficiently small open set containing a steady state $\left(u_{0}, v_{0}\right)$ where $f\left(u_{0}, v_{0}\right)=g\left(u_{0}, v_{0}\right)=0$, assuming that $\nabla f(x) \neq 0$ and $\nabla g(x) \neq 0$ for $x$ sufficiently close to $\left(u_{0}, v_{0}\right) \in \mathbb{R}^{2}$. Under these hypotheses we establish that (simultaneously) all the Cauchy problems attached to (1.7) have a unique solution, with the same maximal interval of existence, see Theorem 7.1 and Lemma 7.1, By using standard techniques based on semigroup theory, one shows the uniqueness of all the initial value problems (1.7), as well as the continuous dependence of the initial conditions. In Section 7.3, we study the Cauchy problem attached to the Brusselator and the CIMA reaction.

In the case $X_{\infty}$, we call the system (1.7) the mean-field model (or approximation) of the original system (1.3). For $M$ sufficiently large, the solution of the Cauchy problem attached to the mean-field model is arbitrarily close to the solution of system (1.7) in $X_{M}$, see Theorem 8.1 .

The matrix $\mathbb{A}^{(M)}$ of the operator $\boldsymbol{L}_{M}$ acting on $X_{M}$ (after renaming the elements of the basis of $X_{M}$ ) is a diagonal-type matrix of the form

$$
\mathbb{A}^{(M)}=\left[\begin{array}{ccccc}
\mathbb{A}_{N ; M} & & & & \\
& \ddots & & & \\
& & \mathbb{A}_{N ; M} & & \\
& & & \ddots & \\
& & & & \mathbb{A}_{N ; M}
\end{array}\right]_{p^{M-N} \times p^{M-N}}
$$

where

$$
\mathbb{A}_{N ; M}=\left[p^{N-M} A_{J I}-\gamma_{I} \delta_{J I}\right]_{J, I \in G_{N}^{0}}=p^{N-M}\left[A_{J I}-p^{M-N} \gamma_{I} \delta_{J I}\right]_{J, I \in G_{N}^{0}}
$$


see Lemma 9.1. The matrix $\mathbb{A}^{(M)}$, which corresponds to a network constructed by using $p^{M-N}$ replicas of the original network, each of these replicas corresponds to a network having a diffusion operator of type $\mathbb{A}_{N ; M}$ and the corresponding $p$-adic diffusion equation is

$$
\frac{\partial f^{(N)}(x, t)}{\partial t}=\varepsilon^{\prime} \boldsymbol{L}_{N, \lambda} f^{(N)}(x, t)
$$

where $\varepsilon^{\prime}=p^{N-M_{\varepsilon}} \varepsilon, \lambda=p^{M-N}$, and $\boldsymbol{L}_{N, \lambda}: X_{N} \rightarrow X_{N}$ is defined as

$$
L_{N, \lambda} \varphi(x)=\int_{\mathcal{K}_{N}}\{\varphi(y)-\lambda \varphi(x)\} J_{N}(x, y) d y .
$$

The equations of type (1.8) form a parametric family indexed by $\left(\varepsilon^{\prime}, \lambda\right)$, which is invariant under a scale change of type $\left(\varepsilon^{\prime}, \lambda\right) \rightarrow\left(\delta \varepsilon^{\prime}, \lambda \delta^{-1}\right)$, for $\delta \in(0,1)$, see Theorems 4.3, 6.2.

In conclusion, the mean-field approximation is the 'limit' of the system (1.7) in $X_{M}$ when $M$ tends to infinity. In turn, any solution of the system (1.7) in $X_{M}(M>N)$ is made of $p^{M-N}$ solutions of $p^{M-N}$ systems of type (1.7) in $X_{N}$. Each of them is a scaled version (a scaled replica) of the original system (1.3).

In order to understand the 'physical contents' of the mean-field model, it is completely necessary to study its diffusion mechanism, which means to study the following Cauchy problem:

$$
\left\{\begin{array}{l}
\frac{\partial f(x, t)}{\partial t}=\varepsilon \boldsymbol{L} f(x, t), \quad x \in \mathcal{K}_{N}, t>0 \\
f(x, 0)=f_{0}(x) \in X_{\infty} .
\end{array}\right.
$$

The semigroup attached to (1.9),$\left\{e^{\varepsilon t \boldsymbol{L}}\right\}_{t \geq 0}$, is a Feller semigroup, and consequently, there is a Markov process attached to (1.9), see Theorem 4.2. This implies that in the mean-field model, the chemical species $u, v$ interact via a random walk like in the classical case (1.1): the chemical reactions involving species $u, v$ occur as a consequence of a random walk of the particles forming them. This random walk is produced by changes in the concentrations, which can be deterministically modeled by Fick's law of diffusion. The whole picture is coded as a $p$-adic heat equation. A similar result holds in $X_{M}$ for the equations (1.9), see Theorem 6.1, and for the equations of type (1.8), see Sections 4.2 and 6.1. It is important to mention here that the $p$-adic diffusion has strong differences with respect to the classical diffusion in $\mathbb{R}$.

There are infinitely many choices for the kernel $J_{N}(x, y)$ such that the corresponding operator $\boldsymbol{L}$ is a diffusion operator, i.e. such that $(1.9)$ is a heat equation, see Theorem 4.2 and the comments following it. Consequently in $X_{\infty}$ there are infinitely many reactiondiffusion systems 'similar' to (1.3).

The $p$-adic heat equations, which include equations of types (1.8) and (1.9), and their associated Markov processes has been studied intensively in the last thirty years due to physical motivations, see e.g. [5], 66, 8], [9], 222, [24], [26], [44], [45], 48], [51] and the references therein. A central paradigm in physics of complex systems (for instance proteins) asserts that the dynamics of such systems can be modeled as a random walk in the energy 
landscape of the system, see e.g. [16], [26], and the references therein. Typically the energy landscape is approximated by an ultrametric space (a tree called the disconnectivity graph of the energy landscape) and a function on this space describing the distribution of the activation barriers, see e.g. [7]. Then the dynamics is described by a discrete system of ODEs on a tree, see e.g. [7], [22], [26], and the references therein.

Avetisov, Kozyrev et al. discovered that under suitable physical and mathematical hypotheses, the above-mentioned discrete system of ODEs on a tree has a 'continuous $p$-adic limit' (a mean-field model) as a $p$-adic diffusion equation, see [5], [6], [22]. From a mathematical perspective, the original system of ODEs on a tree is a good discretization of the corresponding $p$-adic diffusion equation, see [53]. The diffusion in the systems of type (1.7) is $p$-adic diffusion. Our results on $p$-adic diffusion equations are new. Indeed, they correspond to the so-called 'degenerate energy landscapes' in the terminology of Avetisov, Kozyrev et al. In the case in which the kernel $J_{N}$ has the form $J\left(|x-y|_{p}\right)$, the corresponding diffusion equations were studied in [44]. Operators similar to $\boldsymbol{L}$ have been also studied by Bendikov [9], [10] and Kozyrev [25].

Recently, $p$-adic nonlinear reaction-diffusion equations connected with physical models involving tree-like graphs have been studied intensively, see e.g. [3], [4], [19], [20], [21], [34], 35], [52], [53]. An important novelty is that the $p$-adic reaction-diffusion equations presented here are connected with arbitrary (non-oriented, simple) graphs, which means that we are not restricted to tree-like graphs. Since this is a central idea, we discuss it briefly. The dynamics of complex energy landscapes can be approximated by a system of kinetic equations which describe the transitions between local minima energy. The system of kinetic equations has the form

$$
\frac{d}{d t} f_{i}(t)=-\sum_{j} Q_{i, j}\left\{f_{i}(t)-f_{j}(t)\right\}
$$

where $t \in \mathbb{R}$ is time, $f_{i}(t)$ is the population of the $i$-th local minima, $Q=\left[Q_{i, j}\right]$ is the matrix of transition rates. The matrix $Q$ has a special bock structure that can be understood using ultrametrics. The matrix $Q$ admits a $p$-adic parametrization, i.e. $Q_{i, j}=q\left(|l(i)-l(j)|_{p}\right)$ with $l(i) \in p^{m} \mathbb{Z}_{p} / p^{n} \mathbb{Z}_{p}, n \geq m$. By using this $p$-adic parametrization the system (1.10) takes the form

$$
\frac{d}{d t} f_{I}(t)=-\sum_{J \in p^{m} \mathbb{Z}_{p} / p^{n} \mathbb{Z}_{p}} q\left(|I-J|_{p}\right)\left\{f_{I}(t)-f_{J}(t)\right\} p^{-n},
$$

where $I, J \in p^{m} \mathbb{Z}_{p} / p^{n} \mathbb{Z}_{p}$. In the limit $m \rightarrow-\infty, n \rightarrow \infty$, the above system becomes

$$
\frac{\partial}{\partial t} f(x, t)=\int_{\mathbb{Q}_{p}} q\left(|x-y|_{p}\right)\{f(x, t)-f(y, t)\} d y,
$$

where $x, y \in \mathbb{Q}_{p}, t \in \mathbb{R}$, which is a $p$-adic diffusion equation. In the above-mentioned limit process the size of the matrix $Q$ changes. In the limit, the kernel $q$ in (1.11) corresponds to an infinite version of the matrix $Q$. 
In this article the adjacency matrix $\left[A_{J I}\right]_{J, I \in G_{N}^{0}}$ of a (non-oriented, simple) graph plays the role of the matrix $Q$. The adjacency matrix is fixed, in particular the parameter $N$ is fixed. With the matrix $\left[A_{J I}\right]_{J, I \in G_{N}^{0}}$ we construct a kernel $J_{N}(x, y)$, and an operator $\boldsymbol{L}_{N}$, see (1.6). The kernel $J_{N}$ is the analog of $q$, but this kernel is not obtained by a limit process starting with the adjacency matrix. Initially, the operator $\boldsymbol{L}_{N}$ is defined in a finite dimensional $\mathbb{R}$ space $X_{N}$ spanned by the characteristic functions $\left\{\Omega\left(p^{N}|x-I|_{p}\right)\right\}_{I \in G_{N}^{0}}$. By 'refining' the space $X_{N}$, we obtain new function spaces and extensions of the operator $\boldsymbol{L}_{N}$, but all these extensions have the same kernel, $J_{N}(x, y)$. Then the $p$-adic heat equation introduced here are obtained by a limit process on the spaces $X_{N}, N \geq M$, using a fixed kernel $J_{N}(x, y)$. In the previous paragraph, a new $p$-adic diffusion operator, see (1.11), is constructed by a limit process starting with a matrix $Q$. Here, we show that the Laplacian of a graph is a $p$-adic integral operator, which admits an extension to the space of continuous functions supported in a suitable open compact subset of $\mathbb{Q}_{p}$.

Another goal of this work is to study the formation of Turing patterns in the reactiondiffusion systems of type (1.7). The novelty here is the possibility of studying Turing patterns of reaction-diffusion systems on networks by using $p$-adic continuous reaction-diffusion equations. To achieve this goal, it is necessary to understand the spectra of operators $\left[L_{I J}\right]_{I, J \in G_{N}^{0}}, \boldsymbol{L}_{N}, \boldsymbol{L}_{M}$ with $M>N, \boldsymbol{L}$. The spectrum of the graph Laplacian matrix $\left[L_{J I}\right]_{J, I \in G_{N}^{0}}$ is well-understood, see e.g. [47]. The operator $\boldsymbol{L}$ has unique compact extension $\boldsymbol{L}: L^{2}\left(\mathcal{K}_{N}, \mathbb{C}\right) \rightarrow L^{2}\left(\mathcal{K}_{N}, \mathbb{C}\right)$. Furthermore, the space $L^{2}\left(\mathcal{K}_{N}, \mathbb{C}\right)$ has an orthonormal basis formed by eigenfunctions of operator $\boldsymbol{L}$, see Theorem 10.1.

In $X$ • the Turing instability criteria can be established using the classical argument, see Theorem 11.1, Remark 11, and [31, Chapter 2]. In $X_{\infty}$, the Turing pattern has the form

$$
\begin{gathered}
\sum_{\kappa_{1}<\kappa<\kappa_{2}} \sum_{I} A_{I \kappa} e^{\lambda t} \Omega\left(p^{N}|x-I|_{p}\right) \\
+\sum_{\kappa_{1}<\kappa<\kappa_{2}} \sum_{I} \sum_{r n j} B_{r n j} e^{\lambda t} p^{\frac{r}{2}} \cos \left(\left\{p^{-r-1} j x\right\}_{p}\right) \Omega\left(\left|p^{r} x-n\right|_{p}\right) \\
+\sum_{\kappa_{1}<\kappa<\kappa_{2}} \sum_{I} \sum_{r n j} C_{r n j} e^{\lambda t} p^{\frac{r}{2}} \sin \left(\left\{p^{-r-1} j x\right\}_{p}\right) \Omega\left(\left|p^{r} x-n\right|_{p}\right)
\end{gathered}
$$

for $t \rightarrow+\infty$, where $\kappa$ runs through unstable modes, and $\lambda=\lambda(\kappa), r=r(I, \kappa), n=n(I, \kappa)$, and the ball $p^{-r} n+p^{-r} \mathbb{Z}_{p} \subset \mathcal{K}_{N}$. In the case $X_{M}$, with $M \geq N$, the Turing pattern does not contain the terms involving sine and cosine functions. On the other hand, in the results reported in the literature for the Turing patterns on networks the pattern is described as $\sum A_{I} e^{\lambda t} \varphi_{I}$, where $\varphi_{I}$ is the eigenfunction corresponding to $\mu_{I}$, see e.g. [32]. Our results, see Theorems 7.1 and 11.1, show that Turing criteria remain essentially the same as in the classical case. If the Turing instability criteria holds true in $X_{M}$ for some $M \geq N$, then it is true in $X_{\infty}$, but the converse is not true, see 11.1 and Section 11.2.1.

By identifying the ball $I+p^{N} \mathbb{Z}_{p}$ with a cluster, we have that the Turing pattern (1.12) is organized in a finite number of disjoint clusters, each of them supporting a stationary 
pattern. All these patterns are controlled by the same kinetic parameters. Notice that the occurrence of clusters in the Turing patterns is a direct consequence of the hierarchical structure of $\mathbb{Q}_{p}$ : every ball is a finite disjoint union of balls of smaller radii. Notice that this corresponds exactly with the qualitative description of the Turing patterns on networks given by Nakao and Mikhailov [32] in terms of clustering and multistability. We have not found in the current literature results explaining (in a rigorous mathematical way) the qualitative description of the Turing patterns on networks given by Nakao and Mikhailov.

Reaction-diffusion systems on networks result to be ordinary differential equations, under the assumption of Fickian diffusion, and thus standard (local) existence and unicity conditions do apply. This is enough to prove the onset of Turing instability, see e.g. [39, Chapters $6,7]$, [14]. On the other hand, the study of the $p$-adic continuous reaction-diffusion systems on networks and its approximations requires the use of abstract semilinear evolution equations. The study of traveling waves in the $p$-adic continuous reaction-diffusion systems introduced here is an open problem. The classical techniques cannot be applied because the time is a real variable and the position is a $p$-adic number. Nowadays the study of differential equations on graphs is a relevant mathematical matter, see e.g. [11] [30].

In conclusion, we can say that from the perspective of the proposal of Nakao and Mikhailov [32] of studying reaction-diffusion systems on networks and the corresponding Turing patterns using mean-field models, the $p$-adic analysis results to be the natural tool for such studies. The key point is that the clustering and multistability observed in the computer simulations are naturally explained in the $p$-adic continuous model, and to the best of our understanding, these properties cannot be mathematically explained in the original discrete model. Of course, a good discretization of the continuous model drives us to a classical reaction-diffusion system on a network.

\section{BASIC FACTS ON $p$-ADIC ANALYSIS}

In this section we collect some basic results about $p$-adic analysis that will be used in the article. For an in-depth review of the $p$-adic analysis the reader may consult [1], [42], [48].

2.1. The field of $p$-adic numbers. Along this article $p$ will denote a prime number. The field of $p$-adic numbers $\mathbb{Q}_{p}$ is defined as the completion of the field of rational numbers $\mathbb{Q}$ with respect to the $p$-adic norm $|\cdot|_{p}$, which is defined as

$$
|x|_{p}= \begin{cases}0 & \text { if } \quad x=0 \\ p^{-\gamma} & \text { if } \quad x=p^{\gamma} \frac{a}{b}\end{cases}
$$

where $a$ and $b$ are integers coprime with $p$. The integer $\gamma:=\operatorname{ord}(x)$, with $\operatorname{ord}(0):=+\infty$, is called the $p$-adic order of $x$.

Any $p$-adic number $x \neq 0$ has a unique expansion of the form

$$
x=p^{\operatorname{ord}(x)} \sum_{j=0}^{\infty} x_{j} p^{j},
$$


where $x_{j} \in\{0, \ldots, p-1\}$ and $x_{0} \neq 0$. By using this expansion, we define the fractional part of $x \in \mathbb{Q}_{p}$, denoted as $\{x\}_{p}$, to be the rational number

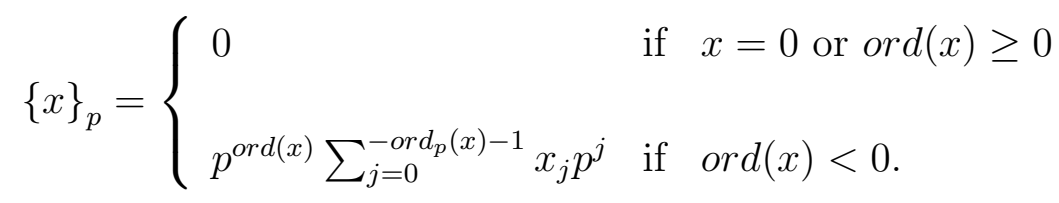

In addition, any non-zero $p$-adic number can be represented uniquely as $x=p^{\text {ord }(x)} a c(x)$ where $a c(x)=\sum_{j=0}^{\infty} x_{j} p^{j}, x_{0} \neq 0$, is called the angular component of $x$. Notice that $|a c(x)|_{p}=1$.

For $r \in \mathbb{Z}$, denote by $B_{r}(a)=\left\{x \in \mathbb{Q}_{p} ;|x-a|_{p} \leq p^{r}\right\}$ the ball of radius $p^{r}$ with center at $a \in \mathbb{Q}_{p}$, and take $B_{r}(0):=B_{r}$. The ball $B_{0}$ equals $\mathbb{Z}_{p}$, the ring of $p$-adic integers of $\mathbb{Q}_{p}$. We also denote by $S_{r}(a)=\left\{x \in \mathbb{Q}_{p} ;|x-a|_{p}=p^{r}\right\}$ the sphere of radius $p^{r}$ with center at $a \in \mathbb{Q}_{p}$, and take $S_{r}(0):=S_{r}$. We notice that $S_{0}=\mathbb{Z}_{p}^{\times}$(the group of units of $\mathbb{Z}_{p}$ ). The balls and spheres are both open and closed subsets in $\mathbb{Q}_{p}$. In addition, two balls in $\mathbb{Q}_{p}$ are either disjoint or one is contained in the other.

The metric space $\left(\mathbb{Q}_{p},|\cdot|_{p}\right)$ is a complete ultrametric space. As a topological space $\left(\mathbb{Q}_{p},|\cdot|_{p}\right)$ is totally disconnected, i.e. the only connected subsets of $\mathbb{Q}_{p}$ are the empty set and the points. In addition, $\mathbb{Q}_{p}$ is homeomorphic to a Cantor-like subset of the real line, see e.g. [1], 48]. A subset of $\mathbb{Q}_{p}$ is compact if and only if it is closed and bounded in $\mathbb{Q}_{p}$, see e.g. [48, Section 1.3], or [1, Section 1.8]. The balls and spheres are compact subsets. Thus $\left(\mathbb{Q}_{p},|\cdot|_{p}\right)$ is a locally compact topological space.

Notation 1. We will use $\Omega\left(p^{-r}|x-a|_{p}\right)$ to denote the characteristic function of the ball $B_{r}(a)$.

2.2. Some function spaces. A complex-valued function $\varphi$ defined on $\mathbb{Q}_{p}$ is called locally constant if for any $x \in \mathbb{Q}_{p}$ there exist an integer $l(x) \in \mathbb{Z}$ such that

$$
\varphi\left(x+x^{\prime}\right)=\varphi(x) \text { for any } x^{\prime} \in B_{l(x)} .
$$

A function $\varphi: \mathbb{Q}_{p} \rightarrow \mathbb{C}$ is called a Bruhat-Schwartz function (or a test function) if it is locally constant with compact support. In this case, we can take $l=l(\varphi)$ in (2.1) independent of $x$, the largest of such integers is called the parameter of local constancy of $\varphi$. The $\mathbb{C}$-vector space of Bruhat-Schwartz functions is denoted by $\mathcal{D}\left(\mathbb{Q}_{p}, \mathbb{C}\right)$. We will denote by $\mathcal{D}\left(\mathbb{Q}_{p}, \mathbb{R}\right)$, the $\mathbb{R}$-vector space of test functions.

Since $\left(\mathbb{Q}_{p},+\right)$ is a locally compact topological group, there exists a Borel measure $d x$, called the Haar measure of $\left(\mathbb{Q}_{p},+\right)$, unique up to multiplication by a positive constant, such that $\int_{U} d x>0$ for every non-empty Borel open set $U \subset \mathbb{Q}_{p}$, and satisfying $\int_{E+z} d x=\int_{E} d x$ for every Borel set $E \subset \mathbb{Q}_{p}$, see e.g. [17, Chapter XI]. If we normalize this measure by the condition $\int_{\mathbb{Z}_{p}} d x=1$, then $d x$ is unique. From now on we denote by $d x$ the normalized Haar measure of $\left(\mathbb{Q}_{p},+\right)$. 
Given $\rho \in[0, \infty)$ and an open subset $U \subset \mathbb{Q}_{p}$, we denote by $L^{\rho}(U, \mathbb{C})$ the $\mathbb{C}$-vector space of all the complex valued functions $g$ defined on $U$ satisfying

$$
\|g\|_{\rho}=\left\{\int_{U}|g(x)|^{\rho} d x\right\}^{\frac{1}{\rho}}<\infty,
$$

and $L^{\infty}(U, \mathbb{C})$ denotes the $\mathbb{C}$-vector space of all the complex valued functions $g$ defined in $U$ such that the essential supremum of $|g|$ is bounded. The corresponding $\mathbb{R}$-vector spaces are denoted as $L^{\rho}(U, \mathbb{R}), 1 \leq \rho \leq \infty$.

Let $U$ be an open subset of $\mathbb{Q}_{p}$, we denote by $\mathcal{D}(U, \mathbb{C})$ the $\mathbb{C}$-vector space of all test functions with support in $U$. For each $\rho \in[1, \infty), \mathcal{D}(U, \mathbb{C})$ is dense in $L^{\rho}(U, \mathbb{C})$, see e.g. [1, Proposition 4.3.3].

2.3. Fourier transform. Set $\chi_{p}(y)=\exp \left(2 \pi i\{y\}_{p}\right)$ for $y \in \mathbb{Q}_{p}$. The map $\chi_{p}$ is an additive character on $\mathbb{Q}_{p}$, i.e. a continuous map from $\left(\mathbb{Q}_{p},+\right)$ into $S$ (the unit circle considered as multiplicative group) satisfying $\chi_{p}\left(x_{0}+x_{1}\right)=\chi_{p}\left(x_{0}\right) \chi_{p}\left(x_{1}\right), x_{0}, x_{1} \in \mathbb{Q}_{p}$. The additive characters of $\mathbb{Q}_{p}$ form an Abelian group which is isomorphic to $\left(\mathbb{Q}_{p},+\right)$, the isomorphism is given by $\xi \rightarrow \chi_{p}(\xi x)$, see e.g. [1, Section 2.3].

If $f \in L^{1}\left(\mathbb{Q}_{p}, \mathbb{C}\right)$ its Fourier transform is defined by

$$
(\mathcal{F} f)(\xi)=\int_{\mathbb{Q}_{p}} \chi_{p}(\xi x) f(x) d x, \quad \text { for } \xi \in \mathbb{Q}_{p} .
$$

We will also use the notation $\mathcal{F}_{x \rightarrow \xi} f$ and $\widehat{f}$ for the Fourier transform of $f$. The Fourier transform is a linear isomorphism (algebraic and topological) from $\mathcal{D}\left(\mathbb{Q}_{p}, \mathbb{C}\right)$ onto itself satisfying

$$
(\mathcal{F}(\mathcal{F} f))(\xi)=f(-\xi)
$$

for every $f \in \mathcal{D}\left(\mathbb{Q}_{p}, \mathbb{C}\right)$, see e.g. [1, Section 4.8]. If $f \in L^{2}$, its Fourier transform is defined as

$$
(\mathcal{F} f)(\xi)=\lim _{k \rightarrow \infty} \int_{|x|_{p} \leq p^{k}} \chi_{p}(\xi x) f(x) d x, \quad \text { for } \xi \in \mathbb{Q}_{p},
$$

where the limit is taken in $L^{2}\left(\mathbb{Q}_{p}, \mathbb{C}\right)$. We recall that the Fourier transform is unitary on $L^{2}\left(\mathbb{Q}_{p}, \mathbb{C}\right)$, i.e. $\|f\|_{2}=\|\mathcal{F} f\|_{2}$ for $f \in L^{2}\left(\mathbb{Q}_{p}, \mathbb{C}\right)$ and that $(2.2)$ is also valid in $L^{2}\left(\mathbb{Q}_{p}, \mathbb{C}\right)$, see e.g. [42, Chapter III, Section 2].

\section{3. $p$-Adic ANALOGUES OF REACTION-DIFFUSION SYSTEMS ON NETWORKS}

We consider an arbitrary graph $\mathcal{G}$ with vertices $I \in G_{N}^{0}$, where $G_{N}^{0}$ is a finite set. Later on we will consider $G_{N}^{0}$ as a subset of $\mathbb{Q}_{p}$, in this way we will obtain an embedding of $\mathcal{G}$ in this space. When there is no connection between the vertices, the dynamics on each vertex is controlled by local interactions described as

$$
\left\{\begin{array}{c}
\frac{\partial u_{J}}{\partial t}=f\left(u_{J}, v_{J}\right) \\
\frac{\partial v_{J}}{\partial t}=g\left(u_{J}, v_{J}\right),
\end{array}\right.
$$


for $J \in G_{N}^{0}$, where a pair $\left(u_{J}, v_{J}\right)=\left(u_{J}(t), v_{J}(t)\right)$ represents some quantities in the vertex $J$, such as population densities of biological species or concentrations of chemical substances. When connection between vertices is taken into account, we assume the existence of a flux of quantities between these vertices. If two vertices are not connected, there is no flux between them. The flux is assumed to be given by Fick's law of diffusion, which means that the flux is proportional to the difference of quantities on the two vertices. Therefore the dynamics of $u_{J}$ and $v_{J}$ on vertex $J$ is described as

$$
\left\{\begin{array}{c}
\frac{\partial u_{J}}{\partial t}=f\left(u_{J}, v_{J}\right)+\varepsilon \sum_{I \in G_{N}^{0}} A_{J I}\left\{u_{I}-u_{J}\right\} \\
\frac{\partial v_{J}}{\partial t}=g\left(u_{J}, v_{J}\right)+\varepsilon d \sum_{I \in G_{N}^{0}} A_{J I}\left\{v_{I}-v_{J}\right\}
\end{array}\right.
$$

for $J \in G_{N}^{0}$, where

$$
A_{J I}:= \begin{cases}1 & \text { if the vertices } J \text { and } I \text { are connected } \\ 0 & \text { otherwise. }\end{cases}
$$

The matrix $\left[A_{J I}\right]_{J, I \in G_{N}^{0}}$ is called the adjacency matrix of $\mathcal{G}$. We admit the possibility that $A_{I I} \neq 0$, which means that graph $\mathcal{G}$ may have self-loops, we also admit the possibility that $A_{I J} \neq A_{J I}$, which means that we do not require that matrix $\left[A_{J I}\right]_{J, I \in G_{N}^{0}}$ be symmetric. However, in Sections 10 and 11, we will require that $\mathcal{G}$ be an undirected graph with a symmetric adjacency matrix.

The positive constants $\varepsilon$ and $\varepsilon d$ denote the diffusivities of $u$ and $v$. The number of edges connecting to vertex $I$ is

$$
\gamma_{I}:=\sum_{J \in G_{N}^{0}} A_{I J}
$$

We set

$$
\gamma_{\mathcal{G}}:=\max _{I \in G_{N}^{0}} \gamma_{I}
$$

For each $J \in G_{N}^{0}$, we can rewrite the flux term as

$$
\sum_{I \in G_{N}^{0}} A_{J I}\left\{u_{I}-u_{J}\right\}=\sum_{I \in G_{N}^{0}} L_{J I} u_{I}
$$

where $L_{J I}=A_{J I}-\gamma_{I} \delta_{J I}$, and $\delta_{J I}$ denotes the Kronecker delta. The matrix $\left[L_{J I}\right]_{J, I \in G_{N}^{0}}$ is called the Laplacian matrix of the graph $\mathcal{G}$. Then, the system (3.2) can be rewritten as

$$
\left\{\begin{array}{l}
\frac{\partial u_{J}}{\partial t}=f\left(u_{J}, v_{J}\right)+\varepsilon \sum_{I \in G_{N}^{0}} L_{J I} u_{I} \\
\frac{\partial v_{J}}{\partial t}=g\left(u_{J}, v_{J}\right)+\varepsilon d \sum_{I \in G_{N}^{0}} L_{J I} v_{I}
\end{array}\right.
$$

for $J \in G_{N}^{0}$. 
3.1. A $p$-Adic analogue of system (3.4). We now construct a $p$-adic analogue of system (3.4). We set

$$
G_{N}:=\mathbb{Z}_{p} / p^{N} \mathbb{Z}_{p} \text { for } N \geq 1
$$

We identify $G_{N}$ with the set of representatives of the form

$$
I=I_{0}+I_{1} p+\ldots+I_{N-1} p^{N-1},
$$

where the $I_{j}$ s are $p$-adic digits. We assume that $G_{N}^{0} \subset G_{N}$. This implies that the number of vertices $\# G_{N}^{0}$ of $\mathcal{G}$ must satisfy $\# G_{N}^{0} \leq p^{N}$. From now on, we fix and $N$ and $p$ such that this inequality holds. There is no a canonical way of choosing $N$ and $p$. On the other hand, since the elements of the form (3.5) belong to $\mathbb{Z}_{p} \backslash p^{N} \mathbb{Z}_{p}$, the assumption $G_{N}^{0} \subset G_{N}$ gives rise an embedding of $\mathcal{G}$ into $\mathbb{Z}_{p} \backslash p^{N} \mathbb{Z}_{p}$.

We define

$$
\mathcal{K}_{N}=\bigsqcup_{I \in G_{N}^{0}} I+p^{N} \mathbb{Z}_{p}
$$

Then $\mathcal{K}_{N}$ is an open compact subset of $\mathbb{Z}_{p}$. We also define

$$
J_{N}(x, y)=p^{N} \sum_{J \in G_{N}^{0}} \sum_{K \in G_{N}^{0}} A_{J K} \Omega\left(p^{N}|x-J|_{p}\right) \Omega\left(p^{N}|y-K|_{p}\right)
$$

$x, y \in \mathbb{Q}_{p}$, where $\left[A_{J I}\right]_{J, I \in G_{N}^{0}}$ is the adjacency matrix of graph $\mathcal{G}$. Notice that $J_{N}(x, y)$ is a test function from $\mathcal{D}\left(\mathcal{K}_{N} \times \mathcal{K}_{N}, \mathbb{R}\right)$.

We denote by $C\left(\mathcal{K}_{N}, \mathbb{R}\right)$ the vector space of all the continuous real-valued functions on $\mathcal{K}_{N}$ endowed with supremum norm, denoted as $\|\cdot\|_{\infty}$. Notice that $C\left(\mathcal{K}_{N}, \mathbb{R}\right)$ is a Banach space. We denote by $X_{N}$, the $\mathbb{R}$-vector space consisting of all the test functions supported in $\mathcal{K}_{N}$ having the form

$$
\varphi(x)=\sum_{J \in G_{N}^{0}} \varphi(J) \Omega\left(p^{N}|x-J|_{p}\right),
$$

where $\varphi(J) \in \mathbb{R}$. We endow $X_{N}$ with the $\|\cdot\|_{\infty}$-norm. Then $X_{N}$ is a closed subspace of $C\left(\mathcal{K}_{N}, \mathbb{R}\right)$, in addition,

$$
X_{N} \simeq\left(\mathbb{R}^{\# G_{N}^{0}},\|\cdot\|_{\infty}\right), \text { as Banach spaces, }
$$

where $\left\|\left(x_{1}, \ldots, x_{\# G_{N}^{0}}\right)\right\|_{\infty}:=\max \left\{\left|x_{1}\right|, \ldots,\left|x_{\# G_{N}^{0}}\right|\right\}$ for $\left(x_{1}, \ldots, x_{\# G_{N}^{0}}\right) \in \mathbb{R}^{\# G_{N}^{0}}$. Notice that

$$
\left\{\Omega\left(p^{N}|x-J|_{p}\right)\right\}_{J \in G_{N}^{0}}
$$

is a basis of $X_{N}$.

We now define the operator

$$
\boldsymbol{L}_{N} \varphi(x)=\int_{\mathcal{K}_{N}}\{\varphi(y)-\varphi(x)\} J_{N}(x, y) d y, \text { for } \varphi \in X_{N} .
$$

Then $\boldsymbol{L}_{N}: X_{N} \rightarrow X_{N}$ is a linear bounded operator satisfying

$$
\left\|\boldsymbol{L}_{N}\right\| \leq 2 \gamma_{\mathcal{G}}
$$


see (3.3). In addition,

$$
\begin{gathered}
\boldsymbol{L}_{N} \Omega\left(p^{N}|x-I|_{p}\right)=\sum_{J \in G_{N}^{0}} A_{J I} \Omega\left(p^{N}|x-J|_{p}\right)-\left(\sum_{K \in G_{N}^{0}} A_{I K}\right) \Omega\left(p^{N}|x-I|_{p}\right) \\
=\sum_{J \in G_{N}^{0}} A_{J I} \Omega\left(p^{N}|x-J|_{p}\right)-\gamma_{I} \Omega\left(p^{N}|x-I|_{p}\right) \\
=\sum_{J \in G_{N}^{0}}\left\{A_{J I}-\gamma_{I} \delta_{J I}\right\} \Omega\left(p^{N}|x-J|_{p}\right) .
\end{gathered}
$$

Consequently, operator $\boldsymbol{L}_{N}: X_{N} \rightarrow X_{N}$ is represented by the matrix

$$
\left[A_{J I}-\gamma_{I} \delta_{J I}\right]_{J, I \in G_{N}^{0}} .
$$

Notation 2. We set $\mathbb{R}_{+}:=[0,+\infty)$.

Consider the following system:

$$
\left\{\begin{array}{l}
u^{(N)}(\cdot, t), v^{(N)}(\cdot, t) \in C^{1}\left(\mathbb{R}_{+}, X_{N}\right) \\
\frac{\partial u^{(N)}(x, t)}{\partial t}=f\left(u^{(N)}(x, t), v^{(N)}(x, t)\right)+\varepsilon \boldsymbol{L}_{N} u^{(N)}(x, t) \\
\frac{\partial v^{(N)}(x, t)}{\partial t}=g\left(u^{(N)}(x, t), v^{(N)}(x, t)\right)+\varepsilon d \boldsymbol{L}_{N} v^{(N)}(x, t) .
\end{array}\right.
$$

By using that

$$
\begin{aligned}
& u^{(N)}(x, t)=\sum_{L \in G_{N}^{0}} u^{(N)}(L, t) \Omega\left(p^{N}|x-L|_{p}\right), \\
& v^{(N)}(x, t)=\sum_{L \in G_{N}^{0}} v^{(N)}(L, t) \Omega\left(p^{N}|x-L|_{p}\right),
\end{aligned}
$$

where $u^{(N)}(L, t), v^{(N)}(L, t)$ belong to $C^{1}\left(\mathbb{R}_{+}\right)$, and (3.7), and the fact that for any $h: \mathbb{R}^{2} \rightarrow$ $\mathbb{R}$,

$$
h\left(u^{(N)}(x, t), v^{(N)}(x, t)\right)=\sum_{L \in G_{N}^{0}} h\left(u^{(N)}(L, t), v^{(N)}(L, t)\right) \Omega\left(p^{N}|x-L|_{p}\right),
$$

we obtain that the system (3.8) can be rewritten as

$$
\left\{\begin{array}{l}
\frac{\partial u^{(N)}(I, t)}{\partial t}= \\
f\left(u^{(N)}(I, t), v^{(N)}(I, t)\right)+\varepsilon \sum_{L \in G_{N}^{0}}\left\{A_{I L}-\gamma_{I} \delta_{I L}\right\} u^{(N)}(L, t) \\
\frac{\partial v^{(N)}(I, t)}{\partial t}= \\
g\left(u^{(N)}(I, t), v^{(N)}(I, t)\right)+\varepsilon d \sum_{L \in G_{N}^{0}}\left\{A_{I L}-\gamma_{I} \delta_{I L}\right\} u^{(N)}(L, t),
\end{array}\right.
$$

for $I \in G_{N}^{0}$, which is exactly the system (3.4). 
In order to obtain a $p$-adic continuous version of the system (3.8), we first notice that for $\varphi \in C\left(\mathcal{K}_{N}, \mathbb{R}\right)$, the function

$$
\boldsymbol{L} \varphi(x)=\int_{\mathcal{K}_{N}}\{\varphi(y)-\varphi(x)\} J_{N}(x, y) d y
$$

belongs to $C\left(\mathcal{K}_{N}, \mathbb{R}\right)$, and that operator $\boldsymbol{L}$ is a linear continuous operator satisfying

$$
\|\boldsymbol{L}\| \leq 2 \gamma_{\mathcal{G}} \text { and } \boldsymbol{L}_{N}=\left.\boldsymbol{L}\right|_{X_{N}} .
$$

By using the fact that operator $\boldsymbol{L}$ is an extension of $\boldsymbol{L}_{N}$, it is natural to postulate that the system

$$
\left\{\begin{array}{l}
u(\cdot, t), v(\cdot, t) \in C^{1}\left(\mathbb{R}_{+}, C\left(\mathcal{K}_{N}, \mathbb{R}\right)\right) \\
\frac{\partial u(x, t)}{\partial t}=f(u, v)+\varepsilon \boldsymbol{L} u(x, t) \\
\frac{\partial v(x, t)}{\partial t}=g(u, v)+\varepsilon d \boldsymbol{L} v(x, t)
\end{array}\right.
$$

is a ' $p$-adic analog' of the system (3.8). In the following sections, we will establish in a mathematically rigorous way this assertion.

\section{4. $p$-ADIC DIFFUSION AND SELF-SIMILARITY IN $X_{\infty}$}

In this section we study the following Cauchy problem:

$$
\left\{\begin{array}{l}
h(x, t) \in C^{1}\left((0, \infty), C\left(\mathcal{K}_{N}, \mathbb{R}\right)\right) \\
\frac{\partial h(x, t)}{\partial t}=\varepsilon \boldsymbol{L} h(x, t), \quad x \in \mathcal{K}_{N}, t>0 \\
h(x, 0)=h_{0}(x) \in C\left(\mathcal{K}_{N}, \mathbb{R}\right)
\end{array}\right.
$$

where $\boldsymbol{L}: C\left(\mathcal{K}_{N}, \mathbb{R}\right) \rightarrow C\left(\mathcal{K}_{N}, \mathbb{R}\right)$ is the operator defined in (3.10). In this section we show that the equation (4.1) is a ' $p$-adic heat equation,' which means that the corresponding semigroup is Feller, and consequently there is a $p$-adic diffusion process in $\mathcal{K}_{N}$ attached to the differential equation (4.1).

4.1. Yosida-Hille-Ray theorem and Feller semigroups. We formulate Yosida-HilleRay Theorem in the setting of $\left(\mathbb{Q}_{p},|\cdot|_{p}\right)$. For a general discussion the reader may consult [15, Chapter 4, Theorem 2.2].

A semigroup $\{\boldsymbol{Q}(t)\}_{t \geq 0}$ on $C\left(\mathcal{K}_{N}, \mathbb{R}\right)$ is said to be positive if $\boldsymbol{Q}(t)$ is a positive operator for each $t \geq 0$, i.e. it maps non-negative functions to non-negative functions. An operator $(\boldsymbol{A}, \operatorname{Dom}(\boldsymbol{A}))$ on $C\left(\mathcal{K}_{N}, \mathbb{R}\right)$ is said to satisfy the positive maximum principle if whenever $h \in \operatorname{Dom}(\boldsymbol{A}) \subseteq C\left(\mathcal{K}_{N}, \mathbb{R}\right), x_{0} \in \mathbb{Q}_{p}$, and $\sup _{x \in \mathbb{Q}_{p}} h(x)=h\left(x_{0}\right) \geq 0$ we have $\boldsymbol{A} h\left(x_{0}\right) \leq 0$.

We recall that every linear operator on $C\left(\mathcal{K}_{N}, \mathbb{R}\right)$ satisfying the positive maximum principle is dissipative, see e.g. [15, Chapter 4, Lemma 2.1]. 
Theorem 4.1 (Hille-Yosida-Ray Theorem). Let $(\boldsymbol{A}, \operatorname{Dom}(\boldsymbol{A}))$ be a linear operator on $C\left(\mathcal{K}_{N}, \mathbb{R}\right)$. The closure $\overline{\boldsymbol{A}}$ of $\boldsymbol{A}$ on $C\left(\mathcal{K}_{N}, \mathbb{R}\right)$ is single-valued and generates a strongly continuous, positive, contraction semigroup $\left\{\boldsymbol{Q}_{t}\right\}_{t \geq 0}$ on $C\left(\mathcal{K}_{N}, \mathbb{R}\right)$ if and only if:

(i) $\operatorname{Dom}(\boldsymbol{A})$ is dense in $C\left(\mathcal{K}_{N}, \mathbb{R}\right)$;

(ii) $\boldsymbol{A}$ satisfies the positive maximum principle;

(iii) $\operatorname{Rank}(\eta \boldsymbol{I}-\boldsymbol{A})$ is dense in $C\left(\mathcal{K}_{N}, \mathbb{R}\right)$ for some $\eta>0$.

Definition 1. A family of bounded linear operators $\boldsymbol{P}_{t}: C\left(\mathcal{K}_{N}, \mathbb{R}\right) \rightarrow C\left(\mathcal{K}_{N}, \mathbb{R}\right)$ is called a Feller semigroup if

(i) $\boldsymbol{P}_{s+t}=\boldsymbol{P}_{s} \boldsymbol{P}_{t}$ and $\boldsymbol{P}_{0}=I$;

(ii) $\lim _{t \rightarrow 0}\left\|\boldsymbol{P}_{t} h-h\right\|_{\infty}=0$ for any $h \in C\left(\mathcal{K}_{N}, \mathbb{R}\right)$;

(iii) $0 \leq \boldsymbol{P}_{t} h \leq 1$ if $0 \leq h \leq 1$, with $h \in C\left(\mathcal{K}_{N}, \mathbb{R}\right)$ and for any $t \geq 0$.

Therefore, Theorem 4.1 characterizes the Feller semigroups. More precisely, if $(\boldsymbol{A}, \operatorname{Dom}(\boldsymbol{A}))$ satisfies Theorem 4.1, then $\boldsymbol{A}$ has a closed extension which is the generator of a Feller semigroup.

Lemma 4.1. The operator $\varepsilon \boldsymbol{L}$ generates a strongly continuous, positive, contraction semigroup $\left\{e^{t \varepsilon \boldsymbol{L}}\right\}_{t \geq 0}$ on $C\left(\mathcal{K}_{N}, \mathbb{R}\right)$.

Proof. We first verify the conditions given in Theorem 4.1. The verification of conditions (i)-(ii) is straightforward. The third condition in Theorem 4.1 is equivalent to the existence of a $\eta>0$ such that for any $h \in C\left(\mathcal{K}_{N}, \mathbb{R}\right)$ the equation

$$
\eta u-\varepsilon \boldsymbol{L} u=h
$$

has a solution $u \in C\left(\mathcal{K}_{N}, \mathbb{R}\right)$. Set

$$
g(x):=\int_{\mathcal{K}_{N}} J_{N}(x, y) d y=\sum_{I \in G_{N}^{0}} \gamma_{I} \Omega\left(p^{N}|x-I|_{p}\right) .
$$

Then $g(x)>0,\|g\|_{\infty} \leq \gamma_{\mathcal{G}}$ and $g \in C\left(\mathcal{K}_{N}, \mathbb{R}\right)$. We now rewrite (4.2) as follows:

$$
u(x)-\varepsilon \int_{\mathcal{K}_{N}} u(y)\left\{\frac{J_{N}(x, y)}{\eta+\varepsilon g(x)}\right\} d y=\frac{h(x)}{\eta+\varepsilon g(x)},
$$

where $\frac{h(x)}{\eta+\varepsilon g(x)} \in C\left(\mathcal{K}_{N}, \mathbb{R}\right)$. Now, the operator $\boldsymbol{T}: C\left(\mathcal{K}_{N}, \mathbb{R}\right) \rightarrow C\left(\mathcal{K}_{N}, \mathbb{R}\right)$ defined as

$$
\boldsymbol{T} u(x)=\varepsilon \int_{\mathcal{K}_{N}} u(y)\left\{\frac{J_{N}(x, y)}{\eta+\varepsilon g(x)}\right\} d y
$$

satisfies $\|\boldsymbol{T}\| \leq \frac{\varepsilon \gamma_{\mathcal{G}}}{\eta}$. By taking $\eta>\varepsilon \gamma_{\mathcal{G}}$, operator $\boldsymbol{I}-\boldsymbol{T}$ has an inverse on $C\left(\mathcal{K}_{N}, \mathbb{R}\right)$. Notice that $\eta$ is independent of $h$.

Therefore $\boldsymbol{L}=\overline{\boldsymbol{L}}$ generates a semigroup $\left\{\boldsymbol{Q}_{t}\right\}_{t \geq 0}$ having the properties announced in Theorem 4.1. On the other hand, since $\varepsilon \boldsymbol{L}$ is a linear bounded operator on a Banach space, $\left\{e^{t \varepsilon \boldsymbol{L}}\right\}_{t \geq 0}$ is a uniformly continuous semigroup, and by using that the infinitesimal generators of $\left\{e^{t \varepsilon \boldsymbol{L}}\right\}_{t \geq 0}$ and $\left\{\boldsymbol{Q}_{t}\right\}_{t \geq 0}$ agree, we have $e^{t \varepsilon \boldsymbol{L}}=\boldsymbol{Q}_{t}$ for $t \geq 0$, see e.g. 38, Theorems 1.2 and $1.3]$. 
Theorem 4.2. There exists a probability measure $p_{t}(x, \cdot), t \geq 0, x \in \mathcal{K}_{N}$, on the Borel $\sigma$-algebra of $\mathcal{K}_{N}$, such that the Cauchy problem (4.1) has a unique solution of the form

$$
h(x, t)=\int_{\mathcal{K}_{N}} h_{0}(y) p_{t}(x, d y) .
$$

In addition, $p_{t}(x, \cdot)$ is the transition function of a Markov process $\mathfrak{X}$ whose paths are right continuous and have no discontinuities other than jumps.

Proof. By Lemma 4.1, $\left\{e^{t \varepsilon \boldsymbol{L}}\right\}_{t \geq 0}$ is a Feller semigroup on $C\left(\mathcal{K}_{N}, \mathbb{R}\right)$. By using the correspondence between Feller semigroups and transition functions, there exists a uniformly stochastically continuous $C_{0}$-transition function $p_{t}(x, d y)$ satisfying condition $(L)$, see [43, Theorem 2.10], such that

$$
e^{t \boldsymbol{L}} h_{0}(x)=\int_{\mathcal{K}_{N}} h_{0}(y) p_{t}(x, d y) \text { for } h_{0} \in C\left(\mathcal{K}_{N}, \mathbb{R}\right),
$$

see e.g. [43, Theorem 2.15]. Now, by using the correspondence between transition functions and Markov processes, there exists a strong Markov process $\mathfrak{X}$ whose paths are right continuous and have no discontinuities other than jumps, see e.g. [43, Theorem 2.12].

4.2. Self-similarity. Theorem 4.2 can be easily extended to a larger class of operators. For instance, take $J(x, y) \in L^{\infty}\left(\mathcal{K}_{N} \times \mathcal{K}_{N}, \mathbb{R}\right), J(x, y) \geq 0$ and $\lambda \geq 1$, and set

$$
\varepsilon L_{\lambda} \varphi(x)=\varepsilon \int_{\mathcal{K}_{N}}\{\varphi(y)-\lambda \varphi(x)\} J(x, y) d y .
$$

Then $L_{\lambda}: C\left(\mathcal{K}_{N}, \mathbb{R}\right) \rightarrow C\left(\mathcal{K}_{N}, \mathbb{R}\right)$ is a linear bounded operator, with $\left\|\varepsilon L_{\lambda}\right\| \leq(1+\lambda) \varepsilon\|J\|_{\infty}$. Notice that condition $\lambda \geq 1$ is essential to assure that operator $\varepsilon L_{\lambda}$ satisfies the positive maximum principle. Theorem 4.2 holds for the following Cauchy problem:

$$
\left\{\begin{array}{l}
h(x, t) \in C^{1}\left((0, \infty), C\left(\mathcal{K}_{N}, \mathbb{R}\right)\right) \\
\frac{\partial h(x, t)}{\partial t}=\varepsilon \boldsymbol{L}_{\lambda} h(x, t), \quad x \in \mathcal{K}_{N}, t>0 \\
h(x, 0)=h_{0}(x) \in C\left(\mathcal{K}_{N}, \mathbb{R}\right) .
\end{array}\right.
$$

Then, for a fixed $J(x, y) \in L^{\infty}\left(\mathcal{K}_{N} \times \mathcal{K}_{N}, \mathbb{R}\right), J(x, y) \geq 0$, (4.4) is a family of $p$-adic diffusion equations parametrized by the set

$$
\mathcal{P}:=\left\{(\varepsilon, \lambda) \in \mathbb{R}_{+}^{2} ; \varepsilon>0, \lambda \geq 1\right\} .
$$

We identify the family (4.4) with the set $\mathcal{P}$. Now, for $\sigma \in(0,1]$, we define the mapping:

$$
\begin{aligned}
S_{\sigma}: \mathcal{P} & \rightarrow \mathcal{P} \\
(\varepsilon, \lambda) & \rightarrow\left(\sigma \varepsilon, \sigma^{-1} \lambda\right) .
\end{aligned}
$$

The set of all $S_{\sigma}$ for $\sigma \in(0,1]$ is naturally a monoid (denoted as $\mathcal{S}_{\mathcal{P}}$ ), under the composition of functions. Therefore, we have established the following result: 
Theorem 4.3. The family $\mathcal{P}$ is invariant under the action of the monoid $\mathcal{S}_{\mathcal{P}}$.

\section{Discretizations}

5.1. Some additional function spaces and operators. Let $M$ be a positive integer satisfying $M \geq N$. We fix a system of representatives $I_{j}$ s for the quotient

$$
G_{I}^{M}:=\left(I+p^{N} \mathbb{Z}_{p}\right) / p^{M} \mathbb{Z}_{p}
$$

This means that

$$
B_{-N}(I)=\bigsqcup_{I_{j} \in G_{I}^{M}} B_{-M}\left(I_{j}\right)
$$

where $B_{-L}(J)=\left\{x \in \mathbb{Q}_{p} ;|x-J|_{p} \leq p^{-L}\right\}$. Now, we set

$$
G_{N}^{M}:=\bigsqcup_{I \in G_{N}^{0}} G_{I}^{M}
$$

Remark 1. Notice that $G_{N}^{N}=G_{N}^{0}$, and thus $G_{N}^{N}$ can be identified with $\mathcal{G}$.

Since $\mathcal{K}_{N}$ is the disjoint union of the $I+p^{N} \mathbb{Z}_{p}$, for $I \in G_{N}^{0}$,

$$
\mathcal{K}_{N}=\bigsqcup_{I \in G_{N}^{0}} \bigsqcup_{I_{j} \in G_{I}^{M}} I_{j}+p^{M} \mathbb{Z}_{p}=\bigsqcup_{I_{j} \in G_{N}^{M}} I_{j}+p^{M} \mathbb{Z}_{p}
$$

We set $X_{M}, M \geq N$, to be the $\mathbb{R}$-vector space of all the test functions supported in $\mathcal{K}_{N}$ of the form

$$
\varphi(x)=\sum_{I_{j} \in G_{N}^{M}} \varphi\left(I_{j}\right) \Omega\left(p^{M}\left|x-I_{j}\right|_{p}\right), \varphi\left(I_{j}\right) \in \mathbb{R},
$$

endowed with the $\|\cdot\|_{\infty}$-norm. This is a real Banach space. For our convenience, from now on, we set $X_{\infty}:=C\left(\mathcal{K}_{N}, \mathbb{R}\right)$ endowed with the $\|\cdot\|_{\infty}$-norm. This is also a real Banach space.

For $M \geq N$, we define $\boldsymbol{P}_{M} \in \mathfrak{B}\left(X_{\infty}, X_{M}\right)$, the bounded linear operators from $X_{\infty}$ into $X_{M}$, as

$$
\boldsymbol{P}_{M} \varphi(x)=\sum_{I_{j} \in G_{N}^{M}} \varphi\left(I_{j}\right) \Omega\left(p^{M}\left|x-I_{j}\right|_{p}\right) .
$$

We denote by $\boldsymbol{E}_{M}: X_{M} \hookrightarrow X_{\infty}, M \geq N$, the natural continuous embedding. Notice that $\left\|\boldsymbol{E}_{M}\right\| \leq 1$, and that $\boldsymbol{P}_{M} \boldsymbol{E}_{M} \varphi=\varphi$ for $\varphi \in X_{M}, M \geq N$.

Notation 3. Whenever this is possible, we will omit in our formulas the operator $\boldsymbol{E}_{M}$, instead we will use the fact that $X_{M} \hookrightarrow X_{\infty}, M \geq N$.

Lemma 5.1. With the above notation, the following assertions hold true: (i) $\left\|\boldsymbol{P}_{M}\right\| \leq 1$; (ii) $\lim _{M \rightarrow \infty}\left\|\boldsymbol{P}_{M} \varphi-\varphi\right\|_{\infty}=0$ for $\varphi \in X_{\infty}$.

Proof. The first part is straightforward. The second part is a consequence of the following two facts: first, $\mathcal{D}\left(\mathcal{K}_{N}, \mathbb{R}\right)$ is dense in $X_{\infty}$ in the $\|\cdot\|_{\infty}$-norm; second, given a function $\varphi \in$ $\mathcal{D}\left(\mathcal{K}_{N}, \mathbb{R}\right)$, there exists an $M$ sufficiently large such that $\varphi \in X_{M}$. Alternatively, the reader may see [53, Lemma 1]. 
We now consider the real Banach spaces $X_{\infty} \oplus X_{\infty}, X_{M} \oplus X_{M}$ for $M \geq N$, endowed with the norm $\|u \oplus v\|:=\max \left\{\|u\|_{\infty},\|v\|_{\infty}\right\}$. We will identify $u \oplus v$ with the column vector $\left[\begin{array}{l}u \\ v\end{array}\right]$.

5.2. Conditions on the nonlinearity. With respect to the nonlinearity we assume the following. We fix $a, b \in \mathbb{R}$, with $a<b$, and assume that

(Hypothesis 1)

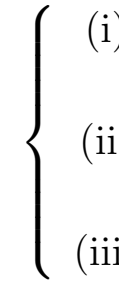

$$
f, g:(a, b) \times(a, b) \rightarrow \mathbb{R}
$$$$
f, g \in C^{1}((a, b) \times(a, b)) ;
$$

(iii) $\nabla f(x, y) \neq 0$ and $\nabla g(x, y) \neq 0$ for any $(x, y) \in(a, b) \times(a, b)$.

Now we define

$$
U=\left\{v \in X_{\infty} ; a<v(x)<b \text { for any } x \in \mathcal{K}_{N}\right\} .
$$

Notice that $U$ is an open set in $X_{\infty}$. Indeed, take $\delta>0$ sufficiently small and $v \in U$, if

$$
h \in B(v, \delta)=\left\{h \in X_{\infty} ;\|v-h\|_{\infty}<\delta\right\},
$$

then

$$
a<-\delta+\min _{x \in \mathcal{K}_{N}} v(x)<h(x)<\delta+\max _{x \in \mathcal{K}_{N}} v(x)<b,
$$

for $\delta$ sufficiently small.

By $\left[\begin{array}{l}f(u, v) \\ g(u, v)\end{array}\right]$, with $u \oplus v \in U \oplus U$, we mean the mapping

$$
\begin{aligned}
{\left[\begin{array}{l}
f \\
g
\end{array}\right]: U \oplus U } & \rightarrow \mathbb{R} \oplus \mathbb{R} \\
u \oplus v & \rightarrow \quad f(u, v) \oplus g(u, v) .
\end{aligned}
$$

5.3. Two Cauchy problems. We denote by $\varepsilon \boldsymbol{L}\left[\begin{array}{ll}1 & 0 \\ 0 & d\end{array}\right]$ the operator acting on $X_{\infty} \oplus X_{\infty}$ as

$$
\varepsilon\left[\begin{array}{ll}
1 & 0 \\
0 & d
\end{array}\right]\left[\begin{array}{l}
u \\
v
\end{array}\right]=\left[\begin{array}{l}
\varepsilon \boldsymbol{L} u \\
\varepsilon d \boldsymbol{L} v
\end{array}\right] .
$$


In the next sections, we will study the following Cauchy problem, for some $\tau>0$ fixed:

$$
\left\{\begin{array}{l}
\frac{\partial}{\partial t}\left[\begin{array}{c}
u(t) \\
v(t)
\end{array}\right]=\left[\begin{array}{c}
f(u(t), v(t)) \\
g(u(t), v(t))
\end{array}\right]+\left[\begin{array}{c}
\varepsilon \boldsymbol{L} u(t) \\
\varepsilon d \boldsymbol{L} v(t)
\end{array}\right] \\
t \in[0, \tau), x \in \mathcal{K}_{N} \\
u(0) \oplus v(0)=u_{0} \oplus v_{0} \in U \oplus U
\end{array}\right.
$$

Also, we will study the Cauchy problem for the following discretization of (5.4), with $\boldsymbol{L}_{M}=$ $\left.\boldsymbol{L}\right|_{X_{M}}$ :

$$
\left\{\begin{array}{l}
\frac{\partial}{\partial t}\left[\begin{array}{c}
u^{(M)}(t) \\
v^{(M)}(t)
\end{array}\right]=\left[\begin{array}{c}
P_{M} f\left(E_{M} u^{(M)}(t), E_{M} v^{(M)}(t)\right) \\
P_{M} g\left(E_{M} u^{(M)}(t), E_{M} v^{(M)}(t)\right)
\end{array}\right]+\left[\begin{array}{c}
\varepsilon \boldsymbol{L}_{M} u^{(M)}(t) \\
\varepsilon d \boldsymbol{L}_{M} v^{(M)}(t)
\end{array}\right], \\
t \in[0, \tau), x \in \mathcal{K}_{N} \\
u^{(M)}(0) \oplus v^{(M)}(0) \in U \cap X_{M} \oplus U \cap X_{M} .
\end{array}\right.
$$

Remark 2. Notice that

$$
\left[\begin{array}{c}
P_{M} f\left(E_{M} u^{(M)}(t), E_{M} v^{(M)}(t)\right) \\
P_{M} g\left(E_{M} u^{(M)}(t), E_{M} v^{(M)}(t)\right)
\end{array}\right]=\left[\begin{array}{c}
f\left(u^{(M)}(t), v^{(M)}(t)\right) \\
g\left(u^{(M)}(t), v^{(M)}(t)\right)
\end{array}\right] .
$$

\section{6. $p$-ADIC DIFFUSION IN $X_{M}$ AND SELF-SIMILARITY}

We first observe that any function $h: G_{N}^{M} \rightarrow \mathbb{R}, I_{j} \rightarrow h\left(I_{j}\right)$ can be uniquely identified with an element of $X_{M}$ :

$$
h(x)=\sum_{I_{j} \in G_{N}^{M}} h\left(I_{j}\right) \Omega\left(p^{M}\left|x-I_{j}\right|_{p}\right), x \in \mathcal{K}_{N} .
$$

In this way, $X_{M}$ is naturally the space of continuous real-valued functions on $G_{N}^{M}$.

Remark 3. Lemma 4.1 holds true in $X_{M}, M \geq N$, i.e. the operator $\varepsilon_{M}$ generates a strongly continuous, positive, contraction semigroup $\left\{e^{t \varepsilon \boldsymbol{L}_{M}}\right\}_{t \geq 0}$ on $X_{M}$.

Now, by Theorem 4.2, $\boldsymbol{L}: X_{\infty} \rightarrow X_{\infty}$ generates a Feller semigroup, since $X_{M} \hookrightarrow X_{\infty}$, $\boldsymbol{L}_{M}=\left.\boldsymbol{L}\right|_{X_{M}}$ and $\boldsymbol{L}_{M}: X_{M} \rightarrow X_{M}$, we have $\boldsymbol{L}_{M}$ generates a Feller semigroup on $X_{M}$ and Theorem 4.2 holds in $X_{M}$ : 
Theorem 6.1. There exists a probability measure $p_{t}^{(M)}(x, \cdot), t \geq 0, x \in \mathcal{K}_{N}$, on the Borel $\sigma$-algebra of $\mathcal{K}_{N}$, such that the Cauchy problem:

$$
\left\{\begin{array}{l}
h^{(M)}(x, t) \in C^{1}\left((0, \infty), X_{M}\right) \\
\frac{\partial h^{(M)}(x, t)}{\partial t}=\varepsilon \boldsymbol{L}_{M} h^{(M)}(x, t), \quad x \in \mathcal{K}_{N}, t>0 \\
h^{(M)}(x, 0)=h_{0}^{(M)}(x) \in X_{M}
\end{array}\right.
$$

has a unique solution of the form

$$
h^{(M)}(x, t)=\int_{\mathcal{K}_{N}} h_{0}^{(M)}(y) p_{t}^{(M)}(x, d y) .
$$

In addition, $p_{t}^{(M)}(x, \cdot)$ is the transition function of a Markov process $\mathfrak{X}^{(M)}$ whose paths are right continuous and have no discontinuities other than jumps.

If $M=N$, then $G_{N}^{M}=G_{N}^{0}$ and Theorem 6.1 describes the diffusion mechanism in $X_{N}$, which is exactly the diffusion mechanism in the original discrete system (1.3). In the construction of the Markov process attached to (6.1) the nature of the points of $G_{N}^{M}$ is not relevant (consequently in the case $M=N$, the embedding of $\mathcal{G}$ into $\mathbb{Q}_{p}$ is not relevant). What matters is the existence of a Feller semigroup acting on a suitable space of continuous functions on $X_{M}$.

6.1. Self-similarity. We take $J(x, y)=J_{N}(x, y)$ and consider operators $L_{\lambda}, \lambda \geq 1$, introduced in Section 4.2, see (4.3). We define, for $M \geq N$,

$$
L_{M, \lambda}=\left.L_{\lambda}\right|_{X_{M}}
$$

This definition makes sense since $X_{M} \hookrightarrow X_{\infty}$. Furthermore, $L_{M, \lambda}: X_{M} \rightarrow X_{M}$ is a bounded linear operator. Theorem 6.1 holds true for the following Cauchy problem:

$$
\left\{\begin{array}{l}
h^{(M)}(x, t) \in C^{1}\left((0, \infty), X_{M}\right) \\
\frac{\partial h^{(M)}(x, t)}{\partial t}=\varepsilon \boldsymbol{L}_{M, \lambda} h^{(M)}(x, t), \quad x \in \mathcal{K}_{N}, t>0 \\
h^{(M)}(x, 0)=h_{0}^{(M)}(x) \in X_{M} .
\end{array}\right.
$$

Thus, (6.3) is a family of $p$-adic diffusion equations on $X_{M}$ parametrized by the set

$$
\mathcal{P}_{M}:=\left\{(\varepsilon, \lambda) \in \mathbb{R}_{+}^{2} ; \varepsilon>0, \lambda \geq 1\right\} .
$$

We identify the family (6.3) with the set $\mathcal{P}_{M}$. By defining $\mathcal{S}_{\mathcal{P}_{M}}$ in an analogous way to $\mathcal{S}_{\mathcal{P}}$ we obtain the following result:

Theorem 6.2. The family $\mathcal{P}_{M}$ is invariant under the action of the monoid $\mathcal{S}_{\mathcal{P}_{M}}$. 
Remark 4. Assume that $(\varepsilon, 1) \in \mathcal{P}_{N}$, i.e. the equation

$$
\frac{\partial h_{I}^{(N)}}{\partial t}=\varepsilon \sum_{J \in G_{N}^{0}}\left\{A_{I J}-\gamma_{I} \delta_{I J}\right\} h_{J}^{(N)}, I \in G_{N}^{0}
$$

is a $p$-adic diffusion equation, then for any $0<\sigma \leq 1,\left(\varepsilon \sigma, \sigma^{-1}\right) \in \mathcal{P}_{N}$, i.e. the equation

$$
\frac{\partial h_{I}^{(N)}}{\partial t}=\varepsilon \sum_{J \in G_{N}^{0}}\left\{\sigma A_{I J}-\gamma_{I} \delta_{I J}\right\} h_{J}^{(N)}, I \in G_{N}^{0}
$$

is a p-adic diffusion equation.

\section{The Cauchy problem in $X$ •}

In this section we study in unified form the Cauchy problems (5.4)-(5.5). We use the following notation:

$$
X_{\bullet}:=\left\{\begin{array}{ll}
X_{\infty} & \text { if } \bullet=\infty \\
X_{M} & \text { if } \bullet=M
\end{array}, \quad \boldsymbol{L}_{\bullet}:= \begin{cases}\boldsymbol{L} & \text { if } \bullet=\infty \\
\boldsymbol{L}_{M} & \text { if } \bullet=M\end{cases}\right.
$$

and $u^{(\bullet)}(t) \oplus v^{(\bullet)}(t)$ means $u(t) \oplus v(t)$ if $\bullet=\infty$. By using this notation and (5.6), the Cauchy problems (5.4)-(5.5), with initial data in $U \cap X_{N} \oplus U \cap X_{N}$, can be written as

$$
\left\{\begin{array}{l}
\frac{\partial}{\partial t}\left[\begin{array}{c}
u^{(\bullet)}(t) \\
v^{(\bullet)}(t)
\end{array}\right]=\left[\begin{array}{c}
f\left(u^{(\bullet)}(t), v^{(\bullet)}(t)\right) \\
g\left(u^{(\bullet)}(t), v^{(\bullet)}(t)\right)
\end{array}\right]+\left[\begin{array}{c}
\varepsilon \boldsymbol{L}_{\bullet} u^{(\bullet)}(t) \\
\varepsilon d \boldsymbol{L}_{\bullet} v^{(\bullet)}(t)
\end{array}\right], \\
t \in[0, \tau), x \in \mathcal{K}_{N} ; \\
u^{(\bullet)}(0) \oplus v^{(\bullet)}(0) \in U \cap X_{N} \oplus U \cap X_{N} .
\end{array}\right.
$$

7.1. Mild solutions. We review the notion of mild solutions for the Cauchy problem (7.1). We will follow [28, Chapter 5]. We use the following conditions:

Condition AS1. $X_{\bullet} \oplus X_{\bullet}$ is a real Banach space.

Condition AS2. The operator $\left[\begin{array}{l}\varepsilon \boldsymbol{L}_{\bullet} \\ \varepsilon d \boldsymbol{L}\end{array}\right]$ is the generator of a strongly continuous semigroup $\left\{e^{\varepsilon t \boldsymbol{L} \bullet}\right\}_{t \geq 0} \oplus\left\{e^{\varepsilon d t \boldsymbol{L} \bullet}\right\}_{t \geq 0}$ satisfying

$$
\left\|e^{\varepsilon t \boldsymbol{L} \bullet} \oplus e^{\varepsilon d t \boldsymbol{L} \bullet}\right\| \leq 1 \text { for } t \geq 0
$$

This condition follows from Lemma 4.1 and Remark 3 , 
Condition AS3. Let $U \subset X_{\infty}$ be the open set defined in (5.2), and let

$$
\left[\begin{array}{l}
f \\
g
\end{array}\right]:(U \oplus U) \rightarrow X_{\infty} \oplus X_{\infty}
$$

be the continuous mapping defined in (5.3). Then for each $u_{0} \oplus v_{0} \in U \oplus U$, there exist $\delta>0$ and $L<\infty$ such that

$$
\left\|\left[\begin{array}{l}
f\left(u_{1}, v_{1}\right) \\
g\left(u_{1}, v_{1}\right)
\end{array}\right]-\left[\begin{array}{c}
f\left(u_{2}, v_{2}\right) \\
g\left(u_{2}, v_{2}\right)
\end{array}\right]\right\| \leq L\left\|\left(u_{1}-u_{2}\right) \oplus\left(v_{1}-v_{2}\right)\right\|,
$$

for $u_{1} \oplus v_{1}, u_{2} \oplus v_{2}$ in the ball $B\left(u_{0} \oplus v_{0}, \delta\right)$. This result follows from the Taylor formula applied to $f$ and $g$ and Hypothesis 1 . Indeed, take $\delta>0$ such that $u_{1} \oplus v_{1}, u_{2} \oplus v_{2} \in$ $B\left(u_{0} \oplus v_{0}, \delta\right) \subset U \oplus U$. This implies

$$
\left\|u_{i}-u_{0}\right\|_{\infty}<\delta \text { and }\left\|v_{i}-v_{0}\right\|_{\infty}<\delta, i=1,2
$$

and by using the definition of $U$,

$$
a<u_{i}<b \text { and } a<v_{i}<b, i=1,2 \text {. }
$$

Now by using the Taylor formula, for $h \in C^{1}((a, b) \times(a, b))$, with $\left(u_{1}, v_{1}\right),\left(u_{1}+h_{1}, v_{1}+h_{2}\right) \in$ $(a, b) \times(a, b)$,

$$
\begin{aligned}
& h\left(u_{1}+h_{1}, v_{1}+h_{2}\right)=h\left(u_{1}, v_{1}\right)+\frac{\partial h\left(u_{1}, v_{1}\right)}{\partial u} h_{1}+\frac{\partial h\left(u_{1}, v_{1}\right)}{\partial v} h_{2} \\
& +\sqrt{h_{1}^{2}+h_{2}^{2}} E\left(\left(u_{1}+h_{1}, v_{1}+h_{2}\right)\right.
\end{aligned}
$$

where $E$ is a continuous function near to $\left(u_{1}, v_{1}\right)$, we obtain

$$
\begin{aligned}
& \left\|f\left(u_{1}, v_{1}\right)-f\left(u_{2}, v_{2}\right)\right\|_{\infty}< \\
& \qquad \delta\left\{\sup _{\substack{a<u_{1}<b \\
<a<v_{1}<b}}\left|\frac{\partial f\left(u_{1}, v_{1}\right)}{\partial u}\right|+\sup _{\substack{a<u_{1}<b \\
<a<v_{1}<b}}\left|\frac{\partial f\left(u_{1}, v_{1}\right)}{\partial v}\right|\right\}+\sqrt{2} \delta \sup _{\substack{a<u_{2}<b \\
<a<v_{2}<b}}\left|E\left(u_{2}, v_{2}\right)\right| .
\end{aligned}
$$

A similar estimation holds for $g\left(u_{1}, v_{1}\right)-g\left(u_{2}, v_{2}\right)$.

Notice that our nonlinearity does not depend on the temporal variable. For this reason, we are using a simpler condition than the one used in [28, Section 5.2].

Remark 5. Take

$$
\left[\begin{array}{l}
f \\
g
\end{array}\right]:\left(U \cap X_{M} \oplus U \cap X_{M}\right) \rightarrow X_{M} \oplus X_{M},
$$

since $X_{M} \hookrightarrow X_{\infty}$, condition (7.2) holds true for map (7.3). 
Definition 2. For $\tau_{0} \in(0, \tau]$, let $\mathcal{S}_{\text {Mild }}\left(\tau_{0}, X_{\bullet} \oplus X_{\bullet}\right)$ be the collection of all $u^{(\bullet)} \oplus v^{(\bullet)} \in$ $C\left(\left[0, \tau_{0}\right), U \cap X_{\bullet} \oplus U \cap X_{\bullet}\right)$ which satisfy

$$
\int_{0}^{t} u^{(\bullet)}(s) d s \in \operatorname{Dom}\left(\varepsilon \boldsymbol{L}_{\bullet}\right)=X_{\bullet} \text { and } \int_{0}^{t} v^{(\bullet)}(s) d s \in \operatorname{Dom}\left(\varepsilon d \boldsymbol{L}_{\bullet}\right)=X_{\bullet},
$$

and

$$
\left\{\begin{array}{l}
u^{(\bullet)}(t)-u^{(\bullet)}(0)+\varepsilon \boldsymbol{L} \bullet \int_{0}^{t} u^{(\bullet)}(s) d s=\int_{0}^{t} f\left(u^{(\bullet)}(s), v^{(\bullet)}(s)\right) d s \\
v^{(\bullet)}(t)-v^{(\bullet)}(0)+\varepsilon d \boldsymbol{L} \bullet \int_{0}^{t} v^{(\bullet)}(s) d s=\int_{0}^{t} g\left(u^{(\bullet)}(s), v^{(\bullet)}(s)\right) d s,
\end{array}\right.
$$

for $t \in\left[0, \tau_{0}\right)$. The elements of $\mathcal{S}_{\text {Mild }}\left(\tau_{0}, X_{\bullet} \oplus X_{\bullet}\right)$ are the called mild solutions of (7.1).

By using well-known results from semigroup theory, see e.g. [28, Theorem 4.7.3], we have $u^{(\bullet)} \oplus v^{(\bullet)} \in \mathcal{S}_{\text {Mild }}\left(\tau_{0}, X_{\bullet} \oplus X_{\bullet}\right)$ if and only if

$$
u^{(\bullet)} \oplus v^{(\bullet)} \in C\left(\left[0, \tau_{0}\right), U \cap X_{\bullet} \oplus U \cap X_{\bullet}\right)
$$

and

$$
\left\{\begin{array}{l}
u^{(\bullet)}(t)=e^{\varepsilon t \boldsymbol{L} \bullet} u^{(\bullet)}(0)+\int_{0}^{t} e^{\varepsilon(t-s) \boldsymbol{L} \bullet} f\left(u^{(\bullet)}(s), v^{(\bullet)}(s)\right) d s \\
v^{(\bullet)}(t)=e^{\varepsilon d t \boldsymbol{L} \bullet} v^{(\bullet)}(0)+\int_{0}^{t} e^{\varepsilon d(t-s) \boldsymbol{L} \bullet} g\left(u^{(\bullet)}(s), v^{(\bullet)}(s)\right) d s
\end{array}\right.
$$

for $t \in\left[0, \tau_{0}\right)$.

The following result shows that Hypothesis 1, which also implies Condition AS3, implies that any mild solution is a classical solution.

Lemma 7.1. $\mathcal{S}_{\text {Mild }}\left(\tau_{0}, X_{\bullet} \oplus X_{\bullet}\right) \subset C^{1}\left(\left[0, \tau_{0}\right), U \cap X_{\bullet} \oplus U \cap X_{\bullet}\right)$.

Proof. Set

$$
\begin{aligned}
r^{(\bullet)}(t) & :=\int_{0}^{t} e^{\varepsilon(t-s) \boldsymbol{L} \bullet} f\left(u^{(\bullet)}(s), v^{(\bullet)}(s)\right) d s \\
s^{(\bullet)}(t) & :=\int_{0}^{t} e^{\varepsilon d(t-s) \boldsymbol{L} \bullet} g\left(u^{(\bullet)}(s), v^{(\bullet)}(s)\right) d s,
\end{aligned}
$$

for $t \in\left[0, \tau_{0}\right)$. Then by (7.4)-(7.5), it is sufficient to show that

$$
r^{(\bullet)}(t), s^{(\bullet)}(t) \in C^{1}\left(\left[0, \tau_{0}\right), U \cap X_{\bullet} \oplus U \cap X_{\bullet}\right) .
$$

We show that

$$
\frac{d}{d t} r^{(\bullet)}(t)=\varepsilon \int_{0}^{t} e^{\varepsilon(t-s) L_{\bullet}} L_{\bullet} f\left(u^{(\bullet)}(s), v^{(\bullet)}(s)\right) d s+f\left(u^{(\bullet)}(t), v^{(\bullet)}(t)\right),
$$

for $t \in\left[0, \tau_{0}\right)$. A similar formula holds for the derivative of $s^{(\bullet)}(t)$. Take $t \in\left[0, \tau_{0}\right)$ and $h \in[0, t-s]$, then

$$
\begin{aligned}
\frac{r^{(\bullet)}(t+h)-r^{(\bullet)}(t)}{h} & =\int_{0}^{t} e^{\varepsilon(t-s) \boldsymbol{L} \bullet}\left\{\frac{e^{\varepsilon h \boldsymbol{L} \bullet}-\boldsymbol{I}}{h}\right\} f\left(u^{(\bullet)}(s), v^{(\bullet)}(s)\right) d s \\
& +\frac{1}{h} \int_{t}^{t+h} e^{\varepsilon(t+h-s) \boldsymbol{L} \bullet} f\left(u^{(\bullet)}(s), v^{(\bullet)}(s)\right) d s .
\end{aligned}
$$


Then

$$
\begin{gathered}
\left\|\frac{r^{(\bullet)}(t+h)-r^{(\bullet)}(t)}{h}-\frac{d}{d t} r^{(\bullet)}(t)\right\|_{\infty} \leq \\
\| \int_{0}^{t} e^{\varepsilon(t-s) \boldsymbol{L} \bullet}\left\{\frac{e^{\varepsilon h \boldsymbol{L} \bullet}-\boldsymbol{I}}{h}-\varepsilon \boldsymbol{L} \bullet f\left(u^{(\bullet)}(s), v^{(\bullet)}(s)\right) d s \|_{\infty}+\right. \\
\| \frac{1}{h} \int_{t}^{t+h} e^{\varepsilon(t-s+h) \boldsymbol{L} \bullet\left\{f\left(u^{(\bullet)}(s), v^{(\bullet)}(s)\right)-f\left(u^{(\bullet)}(t), v^{(\bullet)}(t)\right)\right\} d s \|_{\infty}} \\
=: I_{1}+I_{2} .
\end{gathered}
$$

Now by using that $u^{(\bullet)} \oplus v^{(\bullet)} \in C\left(\left[0, \tau_{0}\right), U \cap X_{\bullet} \oplus U \cap X_{\bullet}\right)$ and Hypothesis 1:

$$
I_{1} \leq\left\|\frac{e^{\varepsilon h \boldsymbol{L} \bullet}-\boldsymbol{I}}{h}-\varepsilon \boldsymbol{L} \bullet\right\| f\left(u^{(\bullet)}(s), v^{(\bullet)}(s)\right)\left\|_{\infty} \leq B\right\| \frac{e^{\varepsilon h \boldsymbol{L} \bullet}-\boldsymbol{I}}{h}-\varepsilon \boldsymbol{L}_{\bullet} \| \rightarrow 0
$$

as $h \rightarrow 0$, where $B=\sup _{u, v \in U \cap X}|f(u, v)|<\infty$. And by using that

$$
u^{(\bullet)} \oplus v^{(\bullet)} \in C\left(\left[0, \tau_{0}\right), U \cap X_{\bullet} \oplus U \cap X_{\bullet}\right)
$$

and Conditions AS2-AS3, we have

$$
\begin{gathered}
I_{2} \leq \frac{1}{h} \int_{t}^{t+h}\left\|f\left(u^{(\bullet)}(s), v^{(\bullet)}(s)\right)-f\left(u^{(\bullet)}(t), v^{(\bullet)}(t)\right)\right\|_{\infty} d s \\
\leq L \frac{1}{h} \int_{t}^{t+h} \max \left\{\left\|u^{(\bullet)}(s)-u^{(\bullet)}(t)\right\|_{\infty},\left\|v^{(\bullet)}(s)-v^{(\bullet)}(t)\right\|_{\infty}\right\} d s \\
\leq L \sup _{s \in[t, t+h]}\left\|u^{(\bullet)}(s)-u^{(\bullet)}(t)\right\|_{\infty}+L \sup _{s \in[t, t+h]}\left\|v^{(\bullet)}(s)-v^{(\bullet)}(t)\right\|_{\infty} \rightarrow 0 \text { as } h \rightarrow 0 .
\end{gathered}
$$

\subsection{Existence for the initial value problems.}

Theorem 7.1. For each $u_{0}^{(\bullet)} \oplus v_{0}^{(\bullet)} \in U \cap X_{N} \oplus U \cap X_{N}$, there exist $\tau_{u_{0}^{(\bullet)} \oplus v_{0}^{(\bullet)}} \in(0, \tau)$ and $u^{(\bullet)} \oplus v^{(\bullet)} \in \mathcal{S}_{\text {Mild }}\left(\tau_{u_{0}^{(\bullet)} \oplus v_{0}^{(\bullet)}}, X \bullet \oplus X.\right)$ such that $u^{(\bullet)}(0) \oplus v^{(\bullet)}(0)=u_{0}^{(\bullet)} \oplus v_{0}^{(\bullet)}$. Furthermore,

$$
\lim _{k \rightarrow \infty} \sup _{0 \leq t \leq \tau_{u_{0}(\bullet) \oplus v_{0}^{(\bullet)}}}\left\|u_{k}^{(\bullet)}(t) \oplus v_{k}^{(\bullet)}(t)-u^{(\bullet)}(t) \oplus v^{(\bullet)}(t)\right\|=0
$$

where $u_{k}^{(\bullet)} \oplus v_{k}^{(\bullet)} \in C\left(\left[0, \tau_{u_{0}^{(\bullet)} \oplus v_{0}^{(\bullet)}}\right], U \cap X \bullet \oplus U \cap X_{\bullet}\right)$ are defined by $u_{1}^{(\bullet)}(t) \oplus v_{1}^{(\bullet)}(t)=u_{0}^{(\bullet)} \oplus v_{0}^{(\bullet)}$ and

$$
\left\{\begin{array}{l}
u_{k+1}^{(\bullet)}(t)=e^{\varepsilon t \boldsymbol{L} \bullet} u_{0}^{(\bullet)}+\int_{0}^{t} e^{\varepsilon(t-s) \boldsymbol{L} \bullet} f\left(u_{k}^{(\bullet)}(s), v_{k}^{(\bullet)}(s)\right) d s \\
v_{k+1}^{(\bullet)}(t)=e^{\varepsilon d t \boldsymbol{L} \bullet} v_{0}^{(\bullet)}+\int_{0}^{t} e^{\varepsilon d(t-s) \boldsymbol{L} \bullet} g\left(u_{k}^{(\bullet)}(s), v_{k}^{(\bullet)}(s)\right) d s
\end{array}\right.
$$

for $t \in\left[0, \tau_{u_{0}^{(\bullet)} \oplus v_{0}^{(\bullet)}}\right]$ and $k \in \mathbb{N} \backslash\{0\}$.

Proof. The results follow from Theorems 5.2.2 and 5.1.2 in [28], by using conditions AS1, AS2, AS3. 
7.2.1. Some additional remarks. The uniqueness of all the initial value problems (7.1) follows from standard results, see e.g. [28, Theorem 5.2.3]. Also, the continuous dependence of the initial value for all the initial value problems (7.1) also holds, see e.g. [28, Theorem 5.2.4].

Maximal interval existence: for $u_{0}^{\bullet} \oplus v_{0}^{\bullet} \in U \cap X_{\bullet} \oplus U \cap X_{\bullet}$, there exists $\tau_{\text {max }}$ depending on the initial datum, and $u^{\bullet} \oplus v^{\bullet} \in \mathcal{S}_{\text {Mild }}\left(\tau_{\max }, X_{\bullet} \oplus X_{\bullet}\right)$ such that $u^{\bullet}(0) \oplus v^{\bullet}(0)=u_{0}^{\bullet} \oplus v_{0}^{\bullet}$, and if $\tau_{\max }<\infty$ then

$$
\int_{0}^{\tau_{\max }}\left\|f\left(u^{\bullet}(t), v^{\bullet}(t)\right)\right\|_{\infty} d t=\infty \text { or } \int_{0}^{\tau_{\max }}\left\|g\left(u^{\bullet}(t), v^{\bullet}(t)\right)\right\|_{\infty} d t=\infty
$$

or there exists $\widetilde{u}^{\bullet} \oplus \widetilde{v}^{\bullet} \in \bar{U} \backslash U$ such that $\lim _{t \rightarrow \tau_{\max }} u^{\bullet}(t) \oplus v^{\bullet}(t)=\widetilde{u}^{\bullet} \oplus \widetilde{v}^{\bullet}$. By taking the same initial datum for all the initial value problems (7.1), we have the same maximal interval existence for all of them.

7.3. Some examples of reaction-diffusion networks on $X_{\bullet}$. When studying Turing instability, one is interested in the study of a system of type (7.1) around a state $u_{0} \oplus v_{0}$ satisfying

$$
f\left(u_{0}, v_{0}\right)=g\left(u_{0}, v_{0}\right)=0 \text { with } u_{0}, v_{0} \text { non-negative real numbers. }
$$

In order to achieve this goal, we pick an open neighborhood $(a, b) \times(a, b) \subset \mathbb{R}^{2}$ containing $\left(u_{0}, v_{0}\right)$, but no other solution of (17.6). Then, by Theorem 7.1, if the initial datum is closed to $\left(u_{0}, v_{0}\right)$, then all the initial value problems (7.1) have mild solutions, and the same maximal interval of existence.

7.3.1. The Brusselator. Taking $A>0$ and $B>0$, the Brusselator on $X$. is the following reaction-diffusion system:

$$
\left\{\begin{array}{l}
u(t), v(t) \in C^{1}\left([0, \tau), X_{\bullet}\right) \\
\frac{\partial u^{(\bullet \bullet}(x, t)}{\partial t}-\varepsilon \boldsymbol{L}_{\bullet} u(x, t)=A-(B+1) u+u^{2} v \\
\frac{\partial v^{(\bullet)}(x, t)}{\partial t}-\varepsilon d \boldsymbol{L}_{\bullet} v(x, t)=B u-u^{2} v
\end{array}\right.
$$

for $t \in[0, \tau), x \in \mathcal{K}_{N}$. This system has only a homogeneous steady state: $u=A, v=\frac{B}{A}$. We consider $f(u, v)=A-(B+1) u+u^{2} v, g(u, v)=B u-u^{2} v$ as functions defined on

$$
(-\delta+A, \delta+A) \times\left(-\delta+\frac{B}{A}, \delta+\frac{B}{A}\right) \subset(a, b) \times(a, b),
$$

for $\delta>0$ sufficiently small. Since $\nabla f(u, v) \neq(0,0)$ and $\nabla g(u, v) \neq(0,0)$ for any $(u, v) \in$ $\mathbb{R} \times \mathbb{R}$, there exist $a, b \in \mathbb{R}$ such that $\left.\nabla f\right|_{(a, b) \times(a, b)} \neq(0,0)$ and $\left.\nabla g\right|_{(a, b) \times(a, b)} \neq(0,0)$, and consequently Hypothesis 1 is valid. Now, we take the subset

$$
\mathcal{U}:=\left\{r \in X_{\infty} ;\|r-A\|_{\infty}<\delta\right\} \oplus\left\{s \in X_{\infty} ;\left\|h-\frac{B}{A}\right\|_{\infty}<\delta\right\} \subset U \oplus U .
$$


Then for any initial datum in $\mathcal{U} \cap X_{\bullet} \oplus X_{\bullet}$, system (7.7) has a unique solution, cf. Theorem 7.1 and Lemma 7.1 .

In the case $X_{\infty}$, the system (17.7) is a $p$-adic version of the Brusselator. This system was first considered by Prigogine and Lefever [40] and Nicolis and Prigogine [33], see also [39, Section 7.5.4].

7.3.2. The CIMA reaction. The CIMA reaction (chlorite-iodide-malonic acid) provided experimental evidence of Turing instability. It was modeled by Lengyel and Epstein [27]. The following is a version of this system in $X_{\bullet}$ :

$$
\left\{\begin{array}{l}
u(t), v(t) \in C^{1}\left([0, \tau), X_{\bullet}\right) \\
\frac{\partial u^{(\bullet)}(x, t)}{\partial t}-\varepsilon \boldsymbol{L}_{\bullet} u(x, t)=A-u-\frac{4 u v}{1+u^{2}} \\
\frac{\partial v^{(\bullet)}(x, t)}{\partial t}-\varepsilon d \boldsymbol{L}_{\bullet} v(x, t)=B C u-\frac{C u v}{1+u^{2}}
\end{array}\right.
$$

for $t \in[0, \tau), x \in \mathcal{K}_{N}$. Here $u$ (the activator) denotes the iodide $\left(I^{-}\right)$concentration and $v$ (the inhibitor) the chlorite $\left(\mathrm{ClO}_{2}^{-}\right)$concentration. We follow the presentation in Perthame's book [39, Section 7.5.2]. Then, we consider this system with $A>0, B>0, C>0$. There is a single homogeneous steady state

$$
u_{0}=\frac{A}{4 B+1}, \quad v_{0}=B\left(1+\frac{A^{2}}{(4 B+1)^{2}}\right) .
$$

We consider $f(u, v)=A-u-\frac{4 u v}{1+u^{2}}, g(u, v)=B C u-\frac{C u v}{1+u^{2}}$ as functions defined in

$$
\left(-\delta+u_{0}, \delta+u_{0}\right) \times\left(-\delta+v_{0}, \delta+v_{0}\right) \subset(a, b) \times(a, b),
$$

for $\delta>0$ sufficiently small so that $(0,0) \notin(a, b) \times(a, b)$. Notice that

$$
\nabla f(u, v)=(0,0) \Leftrightarrow u=0, v=\frac{1}{4} \text { and } \nabla g(u, v)=(0,0) \Leftrightarrow u=0, v=B .
$$

Then, there exist $a, b \in \mathbb{R}$ such that $\left.\nabla f\right|_{(a, b) \times(a, b)} \neq(0,0)$ and $\left.\nabla g\right|_{(a, b) \times(a, b)} \neq(0,0)$, and consequently Hypothesis 1 holds. Now, we take the subset

$$
\mathcal{U}:=\left\{r \in X_{\infty} ;\left\|r-u_{0}\right\|_{\infty}<\delta\right\} \oplus\left\{s \in X_{\infty} ;\left\|h-v_{0}\right\|_{\infty}<\delta\right\} \subset U \oplus U .
$$

Then for any initial datum in $\mathcal{U} \cap X_{\bullet} \oplus X_{\bullet}$, the system (7.8) has a unique solution, cf. Theorem 7.1 and Lemma 7.1. In the case $X_{\infty}$, system (7.8) is a $p$-adic version of the CIMA reaction-diffusion system.

\section{Approximations}

In this section we show that discretization (5.5) provides a good approximation of (5.4). We use standard techniques for approximating nonlinear evolution equations, see e.g. [28]. The following conditions will be needed in order to use the results in [28]: 
8.1. Condition A. (a) $X_{\infty}, X_{N}, X_{N+1}, \ldots$ are real Banach spaces, with the norm $\|u \oplus v\|=$ $\max \left\{\|u\|_{\infty},\|v\|_{\infty}\right\}$.

(b) $\boldsymbol{P}_{M} \oplus \boldsymbol{P}_{M} \in \mathfrak{B}\left(X_{\infty} \oplus X_{\infty}, X_{M} \oplus X_{M}\right)$, for $M \geq N$, where $\boldsymbol{P}_{M}$ as in (5.1). In addition,

$$
\left\|\left(\boldsymbol{P}_{M} \oplus \boldsymbol{P}_{M}\right)(u \oplus v)\right\| \leq\|u \oplus v\| \text { for } M \geq N .
$$

(c) $\boldsymbol{E}_{M} \oplus \boldsymbol{E}_{M} \in \mathfrak{B}\left(X_{\infty} \oplus X_{\infty}, X_{M} \oplus X_{M}\right)$, for $M \geq N$, where $\boldsymbol{E}_{M}$ is the canonical continuous embedding $X_{M} \hookrightarrow X_{\infty}$.

(d) $\left(\boldsymbol{P}_{M} \oplus \boldsymbol{P}_{M}\right)\left(\boldsymbol{E}_{M} \oplus \boldsymbol{E}_{M}\right)(u \oplus v)=u \oplus v$ for $M \geq N$ and $u \oplus v \in X_{M} \oplus X_{M}$.

8.2. Condition B. $\varepsilon \boldsymbol{L}_{M} \oplus \varepsilon d \boldsymbol{L}_{M} \in \mathfrak{B}\left(X_{M} \oplus X_{M}\right)$ for $M \geq N$, and

$$
\left\|e^{\varepsilon t \boldsymbol{L}_{M}} \oplus e^{\varepsilon d t \boldsymbol{L}_{M}}\right\| \leq 1 \text { for } t \geq 0, M \geq N .
$$

This condition follows from Lemma 4.1 and Remark 3. Notice that Condition B, implies that $(-\infty, 0) \subset \rho\left(\varepsilon \boldsymbol{L}_{M}\right) \cap \rho\left(\varepsilon d \boldsymbol{L}_{M}\right)$ for $M \geq N$, where $\rho$ denotes the resolvent set, see e.g. [28, Theorem 4.3.2].

8.3. Condition C'. $\varepsilon \boldsymbol{L} \oplus \varepsilon d \boldsymbol{L}$ is a densely defined linear operator in $X_{\infty} \oplus X_{\infty}, \lambda_{0} \in$ $(-\infty, 0) \cap \rho(\varepsilon \boldsymbol{L}) \cap \rho(\varepsilon d \boldsymbol{L})$ and

$$
\begin{gathered}
\lim _{M \rightarrow \infty} \|\left(\varepsilon \boldsymbol{L}_{M} \oplus \varepsilon d \boldsymbol{L}_{M}\right)\left(\boldsymbol{P}_{M} \oplus \boldsymbol{P}_{M}\right)(u \oplus v)- \\
\left(\boldsymbol{P}_{M} \oplus \boldsymbol{P}_{M}\right)(\varepsilon \boldsymbol{L} \oplus \varepsilon d \boldsymbol{L})(u \oplus v) \|=0,
\end{gathered}
$$

and

$$
\lim _{M \rightarrow \infty}\left\|\left(\boldsymbol{E}_{M} \oplus \boldsymbol{E}_{M}\right)\left(\boldsymbol{P}_{M} \oplus \boldsymbol{P}_{M}\right)(u \oplus v)-(u \oplus v)\right\|=0,
$$

for all $u \oplus v \in \operatorname{Dom}(\varepsilon \boldsymbol{L} \oplus \varepsilon d \boldsymbol{L})$.

Since $\varepsilon \boldsymbol{L} \oplus \varepsilon d \boldsymbol{L}$ is a bounded linear operator $\operatorname{Dom}(\varepsilon \boldsymbol{L} \oplus \varepsilon d \boldsymbol{L})=X_{\infty} \oplus X_{\infty}$ satisfying $\left\|e^{\varepsilon t \boldsymbol{L}} \oplus e^{\varepsilon d t \boldsymbol{L}}\right\| \leq 1$ for $t \geq 0$, the existence of $\lambda_{0}$ is immediate. Condition (8.2) was already established in Lemma 5.1. Condition (8.1) is equivalent to

$$
\lim _{M \rightarrow \infty}\left\|\varepsilon \boldsymbol{L}_{M} \boldsymbol{P}_{M} u-\boldsymbol{P}_{M} \varepsilon \boldsymbol{L} u\right\|_{\infty}=0 \text {, and } \lim _{M \rightarrow \infty}\left\|d \varepsilon \boldsymbol{L}_{M} \boldsymbol{P}_{M} u-\boldsymbol{P}_{M} \varepsilon d \boldsymbol{L} u\right\|_{\infty}=0 .
$$

Now, since $\boldsymbol{L}_{M}=\left.\boldsymbol{L}\right|_{X_{M}}=\boldsymbol{L} \boldsymbol{P}_{M}$, and $\boldsymbol{P}_{M}=\boldsymbol{P}_{M}^{2}$, it is sufficient to show:

Lemma 8.1. $\lim _{M \rightarrow \infty}\left\|\boldsymbol{L}_{M} \boldsymbol{P}_{M} u-\boldsymbol{P}_{M} \boldsymbol{L} u\right\|_{\infty}=0$ for $u \in X_{\infty}$.

Proof. By using the fact that all the operators involved are linear, it is sufficient to establish the lemma for the following class of operators:

$$
\boldsymbol{L}_{J K} u(x):=p^{N} A_{J K} \Omega\left(p^{N}|x-J|_{p}\right) \int_{\mathcal{K}_{N}}\{u(y)-u(x)\} \Omega\left(p^{N}|y-K|_{p}\right) d y,
$$

for $u \in X_{\infty}$. We define

$$
\operatorname{Aver}\left(u ; K+p^{N} \mathbb{Z}_{p}\right)=p^{N} \int_{K+p^{N} \mathbb{Z}_{p}} u(y) d y
$$


Then

$$
\begin{aligned}
\boldsymbol{L}_{J K} u(x)= & \left(A_{J K} \operatorname{Aver}\left(u ; K+p^{N} \mathbb{Z}_{p}\right)\right) \Omega\left(p^{N}|x-J|_{p}\right) \\
& -A_{J K} u(x) \Omega\left(p^{N}|x-J|_{p}\right) .
\end{aligned}
$$

We show that

$$
\lim _{M \rightarrow \infty}\left\|\boldsymbol{L}_{J K} \boldsymbol{P}_{M} u-\boldsymbol{P}_{M} \boldsymbol{L}_{J K} u\right\|_{\infty}=0 \text { for } u \in X_{\infty}
$$

The identity

$$
J+p^{N} \mathbb{Z}_{p}=\bigsqcup_{J_{j} \in G_{J}^{M}} J_{j}+p^{N} \mathbb{Z}_{p}, \text { for } M \geq N
$$

can be rewritten as

$$
\Omega\left(p^{N}|x-J|_{p}\right)=\sum_{J_{j} \in G_{J}^{M}} \Omega\left(p^{N}\left|x-J_{j}\right|_{p}\right), \text { for } M \geq N .
$$

Now by using that

$$
\boldsymbol{P}_{M}\left(\boldsymbol{L}_{J K} u(x)\right)=\sum_{R_{j} \in G_{N}^{M}} \boldsymbol{L}_{J K} u\left(R_{j}\right) \Omega\left(p^{M}\left|x-R_{j}\right|_{p}\right)
$$

and (8.4)-(8.5), we obtain

$$
\begin{gathered}
\boldsymbol{P}_{M}\left(\boldsymbol{L}_{J K} u(x)\right)=\left(A_{J K} \operatorname{Aver}\left(u ; K+p^{N} \mathbb{Z}_{p}\right)\right) \sum_{R_{j} \in G_{J}^{M}} \Omega\left(p^{M}\left|x-R_{j}\right|_{p}\right) \\
-A_{J K} \sum_{R_{j} \in G_{J}^{M}} u\left(R_{j}\right) \Omega\left(p^{M}\left|x-R_{j}\right|_{p}\right),
\end{gathered}
$$

and by using again (8.5) and (8.6), and the definition of $P_{M} u(x)$,

$$
\begin{gathered}
\boldsymbol{P}_{M}\left(\boldsymbol{L}_{J K} u(x)\right)=\left(A_{J K} \operatorname{Aver}\left(u ; K+p^{N} \mathbb{Z}_{p}\right)\right) \Omega\left(p^{N}|x-J|_{p}\right) \\
-A_{J K} \boldsymbol{P}_{M} u(x) \Omega\left(p^{N}|x-J|_{p}\right) .
\end{gathered}
$$

To compute $\boldsymbol{L}_{J K}\left(\boldsymbol{P}_{M} u(x)\right)$, we use first (8.4) to get

$$
\begin{aligned}
\boldsymbol{L}_{J K}\left(\boldsymbol{P}_{M} u(x)\right) & =\left(A_{J K} \operatorname{Aver}\left(\boldsymbol{P}_{M} u ; K+p^{N} \mathbb{Z}_{p}\right)\right) \Omega\left(p^{N}|x-J|_{p}\right) \\
& -A_{J K}\left(\boldsymbol{P}_{M} u(x)\right) \Omega\left(p^{N}|x-J|_{p}\right) .
\end{aligned}
$$

Now from (8.7) and (8.8), we have

$$
\left\|\boldsymbol{P}_{M}\left(\boldsymbol{L}_{J K} u(x)\right)-\boldsymbol{L}_{J K}\left(P_{M} u(x)\right)\right\|_{\infty} \leq A_{J K}\left|\operatorname{Aver}\left(u-\boldsymbol{P}_{M} u ; K+p^{N} \mathbb{Z}_{p}\right)\right| .
$$

On the other hand,

$$
\begin{aligned}
\left|\operatorname{Aver}\left(u-\boldsymbol{P}_{M} u ; K+p^{N} \mathbb{Z}_{p}\right)\right| \leq p^{N} \int_{K+p^{N} \mathbb{Z}_{p}}\left|u(y)-\boldsymbol{P}_{M} u(y)\right| d y \\
\quad \leq \sup _{y \in K+p^{N} \mathbb{Z}_{p}}\left|u(y)-\boldsymbol{P}_{M} u(y)\right| \leq\left\|u-\boldsymbol{P}_{M} u\right\|_{\infty} \rightarrow 0 \text { as } M \rightarrow \infty,
\end{aligned}
$$

cf. Lemma 5.1 . 


\subsection{Existence of good approximations.}

Theorem 8.1. Take $u_{0} \oplus v_{0} \in U \oplus U$. Let $u \oplus v$ be the mild solution of (5.4), and let $u^{(M)} \oplus$ $v^{(M)}$ be the mild solution of (5.5) with initial datum $u^{(M)}(0) \oplus v^{(M)}(0)=\left(P_{M} \oplus P_{M}\right)\left(u_{0} \oplus v_{0}\right)$. Then

$$
\lim _{M \rightarrow \infty} \sup _{0 \leq t \leq \tau}\left\|u^{(M)}(t) \oplus v^{(M)}(t)-u(t) \oplus v(t)\right\|=0
$$

where $\tau<\tau_{\max }$, and $\tau_{\max }$ is the maximal interval of existence for the solution $u(t) \oplus v(t)$ with initial datum $u_{0} \oplus v_{0}$.

Proof. The result follows from Conditions A, B, C', by using the argument given in [28] for Theorem 5.4.7. For this reason, we just provide some details of the proof. Notice that for $t \in[0, \tau], M \geq N$, we have

$$
\begin{aligned}
& \left\{\begin{array}{l}
u^{(M)}(t)=e^{t \varepsilon \boldsymbol{L}_{M}} P_{M} u_{0}+\int_{0}^{t} e^{(t-s) \varepsilon \boldsymbol{L}_{M}} P_{M} f\left(u^{(M)}(s), v^{(M)}(s)\right) d s \\
v^{(M)}(t)=e^{t \varepsilon d \boldsymbol{L}_{M}} P_{M} v_{0}+\int_{0}^{t} e^{(t-s) \varepsilon d \boldsymbol{L}_{M}} P_{M} g\left(u^{(M)}(s), v^{(M)}(s)\right) d s,
\end{array}\right. \\
& \left\{\begin{array}{l}
u(t)=e^{t \varepsilon \boldsymbol{L}} u_{0}+\int_{0}^{t} e^{(t-s) \varepsilon \boldsymbol{L}} f(u(s), v(s)) d s \\
v(t)=e^{t \varepsilon d \boldsymbol{L}} v_{0}+\int_{0}^{t} e^{(t-s) \varepsilon d \boldsymbol{L}} g(u(s), v(s)) d s .
\end{array}\right.
\end{aligned}
$$

The estimations for $\sup _{0 \leq t \leq \tau}\left\|u^{(M)}(t)-u(t)\right\|_{\infty}$ and $\sup _{0 \leq t \leq \tau}\left\|v^{(M)}(t)-v(t)\right\|_{\infty}$ follow from Theorem 5.4.2 in [28], which is a consequence of Conditions A, B, C', by using the reasoning given in the proof Theorem 5.4.7 in [28].

\section{REPLiCAS OF REACTION-DIFFUSION SYSTEMS ON NETWORKS}

In the above section we establish that the $p$-adic continuous model (5.4), which we call the mean-field approximation of the original system (3.9), can be very well approximated by discretization (5.5) for $M$ sufficiently large. The purpose of this section is to study the relations between the solutions of systems (3.9) and (5.5).

We recall that the matrix of operator $\boldsymbol{L}_{N}$ acting on $X_{N}$ is $\left[A_{J I}-\gamma_{I} \delta_{J I}\right]_{J, I \in G_{N}^{0}}$. We set $\mathbb{A}_{N ; M}:=\left[p^{N-M} A_{J I}-\gamma_{I} \delta_{J I}\right]_{J, I \in G_{N}^{0}}$.

Lemma 9.1. The matrix $\mathbb{A}^{(M)}$ of the operator $\boldsymbol{L}_{M}$ acting on $X_{M}$ is a diagonal-type matrix of the form

$$
\mathbb{A}^{(M)}=\left[\begin{array}{ccccc}
\mathbb{A}_{N ; M} & & & & \\
& \ddots & & & \\
& \mathbb{A}_{N ; M} & & \\
& & \ddots & \\
& & & \mathbb{A}_{N ; M}
\end{array}\right]_{p^{M-N} \times p^{M-N}}
$$

after renaming the elements of the basis of $X_{M}$. 
Proof. We first note that kernel $J_{N}(x, y)$, see (3.6), can be rewritten as

$$
\begin{gathered}
J_{N}(x, y)= \\
p^{N} \sum_{J \in G_{N}^{0}} \sum_{K \in G_{N}^{0}} \sum_{J_{j} \in G_{J}^{M}} \sum_{K_{j} \in G_{K}^{M}} A_{J K} \Omega\left(p^{M}\left|x-J_{j}\right|_{p}\right) \Omega\left(p^{M}\left|y-K_{j}\right|_{p}\right),
\end{gathered}
$$

for $(x, y) \in \mathbb{Q}_{p}$. In order to compute the matrix $\mathbb{A}^{(M)}$, it is necessary to compute

$$
\boldsymbol{L}_{M} \Omega\left(p^{M}\left|x-I_{j}\right|_{p}\right) \text { for } I_{j} \in G_{N}^{M}
$$

We first compute

$$
\begin{gathered}
\int_{\mathcal{K}_{N}} \Omega\left(p^{M}\left|y-I_{j}\right|_{p}\right) J_{N}(x, y) d y= \\
p^{N} \sum_{J \in G_{N}^{0}} \sum_{J_{j} \in G_{J}^{M}} A_{J I} \Omega\left(p^{M}\left|x-J_{j}\right|_{p}\right) \int_{\mathcal{K}_{N}} \Omega\left(p^{M}\left|y-I_{j}\right|_{p}\right) d y \\
=p^{N-M} \sum_{J_{j} \in G_{N}^{M}} A_{J I} \Omega\left(p^{M}\left|x-J_{j}\right|_{p}\right) .
\end{gathered}
$$

We now compute

$$
\begin{gathered}
\Omega\left(p^{M}\left|x-I_{j}\right|_{p}\right) \int_{\mathcal{K}_{N}} J_{N}(x, y) d y= \\
p^{N-M}\left\{\sum_{K \in G_{N}^{0} K_{j} \in G_{K}^{M}} A_{I K}\right\} \Omega\left(p^{M}\left|x-I_{j}\right|_{p}\right)= \\
p^{N-M}\left\{\sum_{K \in G_{N}^{0}} p^{M-N} A_{I K}\right\} \Omega\left(p^{M}\left|x-I_{j}\right|_{p}\right)=\gamma_{I} \Omega\left(p^{M}\left|x-I_{j}\right|_{p}\right) .
\end{gathered}
$$

From (9.2)-(9.3) we have

$$
\boldsymbol{L}_{M} \Omega\left(p^{M}\left|x-I_{j}\right|_{p}\right)=\sum_{J_{j} \in G_{N}^{M}}\left[p^{N-M} A_{J I}-\gamma_{I} \delta_{J_{j} I_{j}}\right] \Omega\left(p^{M}\left|x-J_{j}\right|_{p}\right) .
$$

Consequently, the matrix of $\boldsymbol{L}_{M}$ is

$$
\left[p^{N-M} A_{J I}-\gamma_{I} \delta_{J_{j} I_{j}}\right]_{J_{j}, I_{j} \in G_{N}^{M}} .
$$

Given $I \in G_{N}^{0}$, which corresponds to the center of a ball of type $I+p^{N} \mathbb{Z}_{p}$, there are $p^{M-N}$, $I_{j} \mathrm{~s}$ in $G_{I}^{M}$, which correspond to the centers of the balls $I_{j}+p^{M} \mathbb{Z}_{p} \subset I+p^{N} \mathbb{Z}_{p}$, then given any pair $\left(J_{j}, I_{j}\right) \in G_{J}^{M} \times G_{I}^{M}$, the expression $p^{N-M} A_{J I}-\gamma_{I} \delta_{J_{j} I_{j}}$ is constant. More precisely, this value occurs $p^{2(M-N)}$-times when $\left(I_{j}, J_{j}\right)$ runs through $G_{N}^{M} \times G_{N}^{M}$. This implies, that after renaming the elements of the basis of $X_{M}$, the matrix of $\boldsymbol{L}_{M}$ can be rewritten as (9.1).

The matrix $\mathbb{A}^{(M)}$, see (9.1), corresponds to a network constructed by using $p^{M-N}$ replicas of the original network, each of these replicas correspond to a network having a diffusion 
operator of type

$$
\left[p^{N-M} A_{J I}-\gamma_{I} \delta_{J I}\right]_{J, I \in G_{N}^{0}}=p^{N-M}\left[A_{J I}-p^{M-N} \gamma_{I} \delta_{J I}\right]_{J, I \in G_{N}^{0}}
$$

and the corresponding reaction-diffusion equation is

$$
\left\{\begin{array}{l}
\frac{\partial u^{(N)}(x, t)}{\partial t}=f\left(u^{(N)}(x, t), v^{(N)}(x, t)\right)+\varepsilon^{\prime} \boldsymbol{L}_{N, \lambda} u^{(N)}(x, t) \\
\frac{\partial v^{(N)}(x, t)}{\partial t}=g\left(u^{(N)}(x, t), v^{(N)}(x, t)\right)+\varepsilon^{\prime} d \boldsymbol{L}_{N, \lambda} v^{(N)}(x, t),
\end{array}\right.
$$

where $\varepsilon^{\prime}=p^{N-M} \varepsilon, \lambda=p^{M-N}$, where $\boldsymbol{L}_{N, \lambda}: X_{N} \rightarrow X_{N}$ is defined in (6.2). By using the results of Section 6.1 and Remark 4, we say that system (9.5) is a scaled replica of the original system (3.9).

In conclusion, system (5.4) is the 'limit' of system (5.5) when $M$ tends to infinity. This means that both systems have solutions in the time interval $\left[0, \tau_{0}\right)$, and these two solutions are numerically very close for $M$ sufficiently large (Theorem 8.1). In turn, any solution of system (5.5) corresponds to $p^{M-N}$ solutions of $p^{M-N}$ systems of type (9.3), each of them is a scaled version (a replica) of the original system (3.9).

\section{The SPECTRUM OF OPERATOR $\boldsymbol{L}$}

Remark 6. From now on, we assume that $\mathcal{G}$ is an unoriented graph, with a symmetric adjacency matrix $\left[A_{J I}\right]_{J, I \in G_{N}^{0}}$ such that its diagonal contains only zeros. In this case, the spectrum of the discrete Laplacian $\left[L_{J I}\right]_{J, I \in G_{N}^{0}}$ is well-understood, see e.g. [47, Section 4.1]. Since the adjacency matrix $\left[A_{J I}\right]_{J, I \in G_{N}^{0}}$ is symmetric, the eigenvalues, $\mu_{I}, I \in G_{N}^{0}$, of $\left[L_{J I}\right]_{J, I \in G_{N}^{0}}$ are non-positive and $\max _{I \in G_{N}^{0}}\left\{\mu_{I}\right\}=0$. We denote by mult $\left(\mu_{I}\right)$ the multiplicity of the eigenvalue $\mu_{I}$. We set $X_{\bullet} \otimes \mathbb{C}$ for the complexification of $X_{\bullet}$. In particular, $X_{\infty} \otimes \mathbb{C}=C\left(\mathcal{K}_{N}, \mathbb{C}\right)$, with the $L^{\infty}$-norm. Then $\boldsymbol{L}: X_{\infty} \otimes \mathbb{C} \rightarrow X_{\infty} \otimes \mathbb{C}$ is a linear bounded operator. We set $\boldsymbol{L}_{M}:=\left.\boldsymbol{L}\right|_{X_{M} \otimes \mathbb{C}}$.

Lemma 10.1. The operator $\boldsymbol{L}$ has a unique extension to $L^{2}\left(\mathcal{K}_{N}, \mathbb{C}\right)$ as a bounded linear operator.

Proof. We first establish the following assertion:

Claim 1. Let $f \in C\left(\mathcal{K}_{N}, \mathbb{C}\right)$, then $\|\boldsymbol{L} f\|_{2} \leq 2 \sqrt{\sum_{J \in G_{N}^{0}} \gamma_{J}^{2}}\|f\|_{2}$.

By using that

$$
\boldsymbol{L} f(x)=\int_{\mathcal{K}_{N}} f(y) J_{N}(x, y) d y-f(x) \int_{\mathcal{K}_{N}} J_{N}(x, y) d y
$$

we get

$$
\|\boldsymbol{L} f\|_{2} \leq\left\|\int_{\mathcal{K}_{N}} f(y) J_{N}(x, y) d y\right\|_{2}+\left\|f(x) \int_{\mathcal{K}_{N}} J_{N}(x, y) d y\right\|_{2}=: \mathcal{I}_{1}+\mathcal{I}_{2} .
$$


We consider first the term $\mathcal{I}_{1}$. By using Cauchy-Schwarz inequality,

$$
\begin{gathered}
\mathcal{I}_{1}^{2}=\int_{\mathcal{K}_{N}}\left|\int_{\mathcal{K}_{N}} f(y) J_{N}(x, y) d y\right|^{2} d x \leq \int_{\mathcal{K}_{N}}\left\{\int_{\mathcal{K}_{N}}|f(y)| J_{N}(x, y) d y\right\}^{2} d x \\
=\int_{\mathcal{K}_{N}}\left\{p^{N} \sum_{J, I \in G_{N}^{0}} A_{J I} \Omega\left(p^{N}|x-J|_{p}\right) \int_{\mathcal{K}_{N}}|f(y)| \Omega\left(p^{N}|y-I|_{p}\right) d y\right\}^{2} d x \\
\leq \int_{\mathcal{K}_{N}}\left\{\|f\|_{2} p^{\frac{N}{2}} \sum_{J, I \in G_{N}^{0}} A_{J I} \Omega\left(p^{N}|x-J|_{p}\right)\right\}^{2} d x \\
=\|f\|_{2}^{2} p^{N} \sum_{J} \gamma_{J}^{2} \int_{\mathcal{K}_{N}} \Omega\left(p^{N}|x-J|_{p}\right) d x=\|f\|_{2}^{2} \sum_{J \in G_{N}^{0}} \gamma_{J}^{2} .
\end{gathered}
$$

We now consider $\mathcal{I}_{2}$ :

$$
\begin{gathered}
\mathcal{I}_{2}^{2}=\int_{\mathcal{K}_{N}}|f(x)|^{2}\left\{\int_{\mathcal{K}_{N}} J_{N}(x, y) d y\right\}^{2} d x \\
=\int_{\mathcal{K}_{N}}|f(x)|^{2}\left\{\sum_{J \in G_{N}^{0}} \gamma_{J} \Omega\left(p^{N}|x-J|_{p}\right)\right\}^{2} d x \\
=\sum_{J \in G_{N}^{0}} \gamma_{J}^{2} \int_{\mathcal{K}_{N}}|f(x)|^{2} \Omega\left(p^{N}|x-J|_{p}\right) d x \leq\|f\|_{2}^{2} \sum_{J \in G_{N}^{0}} \gamma_{J}^{2} .
\end{gathered}
$$

The announced result follows from Claim 1 , by using the fact that $\mathcal{D}\left(\mathcal{K}_{N}, \mathbb{C}\right) \subset C\left(\mathcal{K}_{N}, \mathbb{C}\right)$ is dense in $L^{2}\left(\mathcal{K}_{N}, \mathbb{C}\right)$, see e.g. [1, Proposition 4.3.3].

Remark 7. The eigenvalues of $\left.\boldsymbol{L}\right|_{X_{N} \otimes \mathbb{C}}=\boldsymbol{L}_{N}$ are exactly the eigenvalues of the matrix $\left[L_{J I}\right]_{J, I \in G_{N}^{0}}$, which are $\mu_{I} \leq 0, I \in G_{N}^{0}$ with $\max _{I \in G_{N}^{0}}\left\{\mu_{I}\right\}=0$. We denote the eigenfunctions of $\left[L_{J I}\right]_{J, I \in G_{N}^{0}}$ as $\varphi_{I}, I \in G_{N}^{0}$.

Let $\left[c_{J}^{I}\right]_{J \in G_{N}^{0}}$ be an eigenvector corresponding to $\mu_{I}$, by identifying it with the function

$$
\varphi_{I}(x):=\sum_{J \in G_{N}^{0}} c_{J}^{I} \Omega\left(p^{N}|x-J|_{p}\right) \in X_{N} \otimes \mathbb{C}, c_{J}^{I} \in \mathbb{C},
$$

and by using that $X_{N} \otimes \mathbb{C} \hookrightarrow X_{\infty} \otimes \mathbb{C}$ and that $\boldsymbol{L}: X_{N} \otimes \mathbb{C} \rightarrow X_{N} \otimes \mathbb{C}$, we have

$$
\left\{\begin{array}{c}
\varphi_{I} \in X_{\infty} \otimes \mathbb{C} \\
\boldsymbol{L} \varphi_{I}=\mu_{I} \varphi_{I}
\end{array}\right.
$$

The $\varphi_{I} s$ form a $\mathbb{C}$-vector space of dimension mult $\left(\mu_{I}\right)$.

Remark 8. We now recall that the set of functions $\left\{\Psi_{r n j}\right\}$ defined as

$$
\Psi_{r n j}(x)=p^{\frac{-r}{2}} \chi_{p}\left(p^{r-1} j x\right) \Omega\left(\left|p^{r} x-n\right|_{p}\right),
$$


where $r \in \mathbb{Z}, j \in\{1, \cdots, p-1\}$, and $n$ runs through a fixed set of representatives of $\mathbb{Q}_{p} / \mathbb{Z}_{p}$, is an orthonormal basis of $L^{2}\left(\mathbb{Q}_{p}\right)$. Furthermore,

$$
\int_{\mathbb{Q}_{p}} \Psi_{r n j}(x) d x=0 .
$$

This result is due to Kozyrev see e.g. [22, Theorem 3.29] or [1, Theorem 9.4.2].

Remark 9. Any function of the the form $\Psi_{r n j}(x)$ supported in $\mathcal{K}_{N}=\bigsqcup_{I \in G_{N}^{0}}\left(I+p^{N} \mathbb{Z}_{p}\right)$ satisfies

$$
\boldsymbol{L} \Psi_{r n j}(x)=-\gamma_{I} \Psi_{r n j}
$$

for some $I \in G_{N}^{0}, j \in\{1, \cdots, p-1\}$.

Theorem 10.1. The elements of the set:

$$
\left\{\mu_{I} ; I \in G_{N}^{0} \backslash\left\{I_{0}\right\}\right\} \bigsqcup\left\{-\gamma_{I} ; I \in G_{N}^{0}\right\} \subset(-\infty, 0)
$$

where $\left\{\mu_{I}\right\}_{I \in G_{N}^{0} \backslash\left\{I_{0}\right\}}$ are the non-zero eigenvalues of matrix $\left[L_{J I}\right]_{J, I \in G_{N}^{0}}$, are the non-zero eigenvalues of $\boldsymbol{L}$. The corresponding eigenfunctions are

$$
\left\{\frac{\varphi_{I}}{\left\|\varphi_{I}\right\|_{2}} ; I \in G_{N}^{0}\right\} \bigsqcup\left\{\Psi_{r n j} ; \operatorname{supp} \Psi_{r n j} \subset \mathcal{K}_{N}\right\},
$$

where the functions $\varphi_{I}, \Psi r n j$ are defined in Remarks [9, 9, respectively. Furthermore, the set 10.4) is an orthonormal basis of $L^{2}\left(\mathcal{K}_{N}, \mathbb{C}\right)$, and

$$
L^{2}\left(\mathcal{K}_{N}, \mathbb{C}\right)=X_{N} \otimes \mathbb{C} \oplus \mathcal{L}_{0}^{2}\left(\mathcal{K}_{N}, \mathbb{C}\right)
$$

where

$$
\mathcal{L}_{0}^{2}\left(\mathcal{K}_{N}, \mathbb{C}\right):=\left\{f \in L^{2}\left(\mathcal{K}_{N}, \mathbb{C}\right) ; \int_{\mathcal{K}_{N}} f d x=0\right\} .
$$

Proof. By Remarks (7), (9), it is sufficient to show (10.5). This assertion follows from Propositions 1, 2 in [54].

\section{Turing Instability}

11.1. Turing Criteria. We now consider a homogeneous steady state $\left(u_{0}, v_{0}\right)$ of (5.4), which is a nonnegative solution of

$$
f(u, v)=g(u, v)=0 .
$$

Since $u, v$ are real-valued functions, to study the linear stability of $\left(u_{0}, v_{0}\right)$, we can use the classical results. Following Turing, in the absence of any spatial variation, the homogeneous state must be linearly stable. With no spatial variation $u, v$ satisfy

$$
\left\{\begin{array}{c}
\frac{\partial u}{\partial t}(x, t)=f(u, v) \\
\frac{\partial v}{\partial t}(x, t)=g(u, v) .
\end{array}\right.
$$

Notice that (11.2) is an ordinary system of differential equations in $\mathbb{R}^{2}$. 
Now, for $\delta>0$ sufficiently small and $\left(u_{0}, v_{0}\right)$ as in (11.1), we define

$$
\begin{aligned}
& U_{\delta, u_{0}} \oplus U_{\delta, v_{0}}= \\
& \left\{u_{1} \oplus u_{2} \in C\left(\mathcal{K}_{N}, \mathbb{R}\right) \oplus C\left(\mathcal{K}_{N}, \mathbb{R}\right) ;\left\|u_{1}-u_{0}\right\|_{\infty}<\delta,\left\|v_{1}-v_{0}\right\|_{\infty}<\delta\right\} .
\end{aligned}
$$

Then, the Cauchy problem:

$$
\left\{\begin{array}{l}
u \oplus v \in C^{1}\left(\left[0, \tau_{0}\right), U_{\delta, u_{0}} \oplus U_{\delta, v_{0}}\right) \\
\frac{\partial}{\partial t}\left[\begin{array}{c}
u(t) \\
v(t)
\end{array}\right]=\left[\begin{array}{c}
f(u(t), v(t)) \\
g(u(t), v(t))
\end{array}\right]+\varepsilon \boldsymbol{L D}\left[\begin{array}{c}
u(t) \\
v(t)
\end{array}\right] ; \\
u(0) \oplus v(0) \in U_{\delta, u_{0}} \oplus U_{\delta, v_{0}},
\end{array}\right.
$$

where

$$
\mathbb{D}=\left[\begin{array}{ll}
1 & 0 \\
0 & d
\end{array}\right]
$$

has a classical solution, cf. Theorem 7.1 and Lemma 7.1. Our goal is to give an asymptotic profile as $t$ tends infinity of this mild solution (the Turing instability criteria). We linearize system (11.3) about the steady state $\left(u_{0}, v_{0}\right)$, by setting

$$
\boldsymbol{w}=\left[\begin{array}{l}
w_{1} \\
w_{2}
\end{array}\right]=\left[\begin{array}{c}
u-u_{0} \\
v-v_{0}
\end{array}\right]
$$

By using the fact that $f$ and $g$ are differentiable, and assuming that $\|\boldsymbol{w}\|=\left\|w_{1} \oplus w_{2}\right\|$ is sufficiently small, then (11.2) can be approximated as

$$
\frac{\partial \boldsymbol{w}}{\partial t}(x, t)=\mathbb{J} \boldsymbol{w}
$$

where

$$
\mathbb{J}_{u_{0}, v_{0}}=: \mathbb{J}=\left[\begin{array}{cc}
\frac{\partial f}{\partial u} & \frac{\partial f}{\partial v} \\
\frac{\partial g}{\partial u} & \frac{\partial g}{\partial v}
\end{array}\right]\left(u_{0}, v_{0}\right)=:\left[\begin{array}{cc}
f_{u_{0}} & f_{v_{0}} \\
g_{u_{0}} & g_{v_{0}}
\end{array}\right] .
$$

We now look for solutions of (11.5) of the form

$$
\boldsymbol{w}(t ; \lambda)=e^{\lambda t} \boldsymbol{w}_{0}
$$

By substituting (11.6) in (11.5), the eigenvalues $\lambda$ are the solutions of

$$
\operatorname{det}(\mathbb{J}-\lambda \mathbb{I})=0,
$$

i.e.

$$
\lambda^{2}-(\operatorname{Tr} \mathbb{J}) \lambda+\operatorname{det} \mathbb{J}=0
$$


Consequently

$$
\lambda=\frac{1}{2}\left\{\operatorname{Tr} \mathbb{J} \pm \sqrt{(\operatorname{Tr} \mathbb{J})^{2}-4 \operatorname{det} \mathbb{J}}\right\} .
$$

The steady state $\boldsymbol{w}=\mathbf{0}$ is linearly stable if $\operatorname{Re} \lambda<0$, this last condition is guaranteed if

$$
\operatorname{Tr} \mathbb{J}<0 \text { and } \operatorname{det} \mathbb{J}>0 .
$$

We now consider the full reaction-ultradiffusion system (11.3). We linearize it about the steady state, which with (11.4) is $\boldsymbol{w}=\mathbf{0}:=\left[\begin{array}{l}0 \\ 0\end{array}\right]$, to get

$$
\left\{\begin{array}{l}
u \oplus v \in C^{1}\left([0, \tau), U_{\delta, u_{0}} \oplus U_{\delta, v_{0}}\right) \\
\frac{\partial}{\partial t} \boldsymbol{w}(x, t)=(\mathbb{J}+\varepsilon \boldsymbol{L D}) \boldsymbol{w}(x, t), t \in[0, \tau) \\
u(0) \oplus v(0) \in U_{\delta, u_{0}} \oplus U_{\delta, v_{0}},
\end{array}\right.
$$

where $\mathbb{J}+\varepsilon \boldsymbol{L} \mathbb{D}$ is a strongly continuous semigroup on $C\left(\mathcal{K}_{N}, \mathbb{R}\right) \oplus C\left(\mathcal{K}_{N}, \mathbb{R}\right)$, see e.g. [28, Corollary 5.1.3], then (11.10) has a mild solution, which is differentiable and unique, see e.g. [28, Theorems 5.1.2, 4.3.1]. Furthermore, (11.10), has also a unique solution, when $\boldsymbol{L}$ is considered as an operator on $L^{2}\left(\mathcal{K}_{N}, \mathbb{C}\right)$, cf. Lemma 10.1, for this reason, we can use the orthonormal basis given in Theorem 10.1 to solve (11.10) in $L^{2}\left(\mathcal{K}_{N}, \mathbb{C}\right)$, by using the separation of variables method, then, the solution of the original problem is exactly the real part of the solution of (11.10) in $L^{2}\left(\mathcal{K}_{N}, \mathbb{C}\right)$.

To solve the system (11.10) in $L^{2}\left(\mathcal{K}_{N}, \mathbb{C}\right)$, we first consider the following eigenvalue problem:

$$
\left\{\begin{array}{l}
\boldsymbol{L D} \boldsymbol{w}_{\kappa}(x)=\kappa \boldsymbol{w}_{\kappa}(x) \\
\boldsymbol{w}_{\kappa} \in L^{2}\left(\mathcal{K}_{N}, \mathbb{C}\right) \oplus L^{2}\left(\mathcal{K}_{N}, \mathbb{C}\right),
\end{array}\right.
$$

which has a solution $\boldsymbol{w}_{\kappa}=w_{\kappa, 1} \oplus w_{\kappa, 2}$ due to Theorem 10.1, where

$$
w_{\kappa, 1}, w_{\kappa, 2} \in\left\{\frac{\varphi_{I}}{\left\|\varphi_{I}\right\|_{2}} ; I \in G_{N}^{0}\right\} \bigsqcup\left\{\Psi_{r n j} ; \operatorname{supp} \Psi_{r n j} \subset \mathcal{K}_{N}\right\} .
$$

We look for an solution of type

$$
\boldsymbol{w}(x, t)=\sum_{r n j} \boldsymbol{a}_{r n j} e^{\lambda t} \Psi_{r n j}+\sum_{I \in G_{N}^{0}} \boldsymbol{b}_{I} \varphi_{I}
$$

where the vectors $\boldsymbol{a}_{r n j}, \boldsymbol{b}_{I}$ are determined by the Fourier expansion of the initial conditions. Substituting (11.12) with (11.11) in (11.10), we obtain that the existence of a non-trivial solution $\boldsymbol{w}(x, t)$ requires that the $\lambda$ s satisfy

$$
\operatorname{det}(\lambda \mathbb{I}-\mathbb{J}-\varepsilon \kappa \mathbb{D})=0,
$$


i.e.,

$$
\lambda^{2}-\{(1+d) \varepsilon \kappa+\operatorname{Tr} \mathbb{J}\} \lambda+h(\kappa)=0,
$$

where

$$
h(\kappa):=\varepsilon^{2} d \kappa^{2}+\varepsilon \kappa\left(d f_{u_{0}}+g_{v_{0}}\right)+\operatorname{det} \mathbb{J} .
$$

Notice that condition (11.13) becomes condition (11.7) when $\kappa=0$. By using the classical reasoning, see e.g. [31, Chapter 2], one obtains the necessary conditions (T1)-(T4) given in Theorem 11.1.

The analysis of the sufficient conditions is similar to the classical case, see e.g. [31, Chapter $2]$. One shows that the condition $h(\kappa)<0$, for some $\kappa \neq 0$, requires that

$$
\frac{\left(d f_{u_{0}}+g_{v_{0}}\right)^{2}}{4 d}>\operatorname{det} \mathbb{J} \text {. }
$$

Furthermore, there exists a critical diffusion $d_{c}$, which is given as an appropriate root of

$$
f_{u_{0}}^{2} d_{c}^{2}+2\left(2 f_{v_{0}} g_{u_{0}}-f_{u_{0}} g_{v_{0}}\right) d_{c}+g_{v_{0}}^{2}=0 .
$$

For $d>d_{c}$ model $((11.3))$ exhibits Turing instability, while for $d<d_{c}$ no. When $d>d_{c}$, there exists a range of unstable of positive wavenumbers $\kappa_{1}<\kappa<\kappa_{2}$, where $\kappa_{1}$, $\kappa_{2}$ are the zeros of $h(\kappa)=0$, see (11.15) and (11.16):

$$
\begin{aligned}
& \kappa_{2}=\frac{-1}{2 d \varepsilon}\left\{\left(d f_{u_{0}}+g_{v_{0}}\right)-\sqrt{\left(d f_{u_{0}}+g_{v_{0}}\right)^{2}-4 d \operatorname{det} \mathbb{J}}\right\}<0, \\
& \kappa_{1}=\frac{-1}{2 d \varepsilon}\left\{\left(d f_{u_{0}}+g_{v_{0}}\right)+\sqrt{\left(d f_{u_{0}}+g_{v_{0}}\right)^{2}-4 d \operatorname{det} \mathbb{J}}\right\}<0 .
\end{aligned}
$$

Notice that, within the unstable range, $\operatorname{Re} \lambda(\kappa)>0$ has a maximum for the wavenumber $\kappa_{\min }=\frac{-\left(d f_{u_{0}}+g_{v_{0}}\right)}{2 \varepsilon d_{c}}$.

In the solution $\boldsymbol{w}(x, t)$ given by (11.12), the dominant contributions as $t$ increases are the modes for which $\operatorname{Re} \lambda(\kappa)>0$ since the other modes tend to zero exponentially, thus, if

$$
\left\{\kappa \in \sigma(L) \backslash\{0\} ; \kappa_{1}<\kappa<\kappa_{2}\right\} \neq \emptyset,
$$

then

$$
\begin{gathered}
\boldsymbol{w}(x, t) \sim \sum_{\kappa_{1}<\kappa<\kappa_{2}} \sum_{I} A_{I \kappa} e^{\lambda t} \Omega\left(p^{N}|x-I|_{p}\right)+ \\
\sum_{\kappa_{1}<\kappa<\kappa_{2}} \sum_{I} \sum_{r n j} B_{r n j} e^{\lambda t} p^{\frac{r}{2}} \cos \left(\left\{p^{-r-1} j x\right\}_{p}\right) \Omega\left(p^{r}|x-I|_{p}\right)+ \\
\sum_{\kappa_{1}<\kappa<\kappa_{2}} \sum_{I} \sum_{r n j} C_{r n j} e^{\lambda t} p^{\frac{r}{2}} \sin \left(\left\{p^{-r-1} j x\right\}_{p}\right) \Omega\left(p^{r}|x-I|_{p}\right)
\end{gathered}
$$

for $t \rightarrow+\infty$, where $\lambda=\lambda(\kappa), r=r(I, \kappa), n=(I, \kappa)$.

Expansion (11.18) implies that

$$
\int_{0}^{\tau}\|f(u(t), v(t))\|_{\infty} d t<\infty \text { and } \int_{0}^{\tau}\|g(u(t), v(t))\|_{\infty} d t<\infty
$$


for any $\boldsymbol{\tau}<\infty$. Which implies that $\tau_{\max }=+\infty$, for any initial data in $U_{\delta, u_{0}} \oplus U_{\delta, v_{0}}$. Notice that in (11.19), we can replace $\|\cdot\|_{\infty}$ by $\|\cdot\|_{2}$. We now formulate the Turing instability criteria for our $p$-adic continuous model.

Remark 10. In the case of reaction-diffusion systems on $X_{M}, M \geq N$, the Turing pattern (11.18) does not contain the sine and cosine functions.

Theorem 11.1. Consider the reaction-diffusion system (11.10). The steady state $\left(u_{0}, v_{0}\right)$ is linearly unstable (Turing unstable) if the following conditions hold:

(T1) $\operatorname{Tr} \mathbb{J}=f_{u_{0}}+g_{v_{0}}<0$;

(T2) $\operatorname{det} \mathbb{J}=f_{u_{0}} g_{v_{0}}-f_{v_{0}} g_{u_{0}}>0$;

(T3) $d f_{u_{0}}+g_{v_{0}}>0$;

(T4) the derivatives $f_{u_{0}}$ and $g_{v_{0}}$ must have opposite signs;

(T5) $\left(d f_{u_{0}}+g_{v_{0}}\right)^{2}-4 d\left(f_{u_{0}} g_{v_{0}}-f_{v_{0}} g_{u_{0}}\right)>0$;

(T6) $\left\{\kappa \in \sigma(\boldsymbol{L}) \backslash\{0\} ; \kappa_{1}<\kappa<\kappa_{2}\right\} \neq \emptyset$;

Furthermore in (11.3), we can take $\tau_{0}=+\infty$, for any initial data in $U_{\delta, u_{0}} \oplus U_{\delta, v_{0}}$.

Remark 11. Theorem 11.1 is also valid for reaction-diffusion systems on $X_{M}$, for $M \geq N$. In this case condition (T6) should be replaced by $\left\{\kappa \in \sigma\left(\boldsymbol{L}_{M}\right) \backslash\{0\} ; \kappa_{1}<\kappa<\kappa_{2}\right\} \neq \emptyset$. Now since

$$
\left\{\kappa \in \sigma\left(\boldsymbol{L}_{M}\right) \backslash\{0\} ; \kappa_{1}<\kappa<\kappa_{2}\right\} \subset\left\{\kappa \in \sigma(\boldsymbol{L}) \backslash\{0\} ; \kappa_{1}<\kappa<\kappa_{2}\right\},
$$

the existence of Turing patterns in $X_{M}$ implies the existence of Turing patterns in $X_{\infty}$, but the converse is not necessarily true such as the examples presented in the next section show.

11.2. Turing patterns for the $p$-adic Brusselator. In this section, we study the existence of Turing patterns for the $p$-adic Brusselator in $X_{M}$, see Section 7.3.1. There is only a homogeneous steady state $u_{0}=A, v_{0}=\frac{B}{A}$, the Jacobian matrix of $(f, g)$ at $\left(u_{0}, v_{0}\right)$ is

$$
\mathbb{J}=\left[\begin{array}{ll}
B-1 & A^{2} \\
-B & -A^{2}
\end{array}\right],
$$

and thus $\operatorname{Tr} \mathbb{J}=B-1-A^{2}$ and $\operatorname{det} \mathbb{J}=A^{2}$. Condition (T1) in Theorem 11.1 implies that

$$
B<1+A^{2}
$$

The conditions (T2) holds true immediately. By taking $B>1$, condition (T4) holds true. Condition (T3) implies that

$$
B>\frac{A^{2}}{d}+1
$$

Notice that $d>1$. Condition (T6) requires that

$$
\left(d(B-1)-A^{2}\right)^{2}-4 d A^{2}>0 \Leftrightarrow d(B-1)>A^{2}+2 \sqrt{d} A .
$$


To verify condition (T6), we compute $\kappa_{1}, \kappa_{2}$ as

$$
\begin{aligned}
\kappa_{2,1} & =\frac{-1}{2 d \varepsilon}\left\{\left(d f_{u_{0}}+g_{v_{0}}\right) \mp \sqrt{\left(d f_{u_{0}}+g_{v_{0}}\right)^{2}-4 d \text { det } \mathbb{J}}\right\} \\
& =\frac{1}{2 d \varepsilon}\left\{\left(A^{2}-d(B-1)\right) \pm \sqrt{\left(A^{2}-d(B-1)\right)^{2}-4 d A^{2}}\right\} \\
& =\frac{1}{2 d \varepsilon}\left\{\left(A^{2}-d(B-1)\right) \mp\left(A^{2}-d(B-1)\right) \sqrt{1-\frac{4 d A^{2}}{\left(A^{2}-d(B-1)\right)^{2}}}\right\} \\
& =\frac{\left(A^{2}-d(B-1)\right)}{2 d \varepsilon}\left\{1 \mp \sqrt{\left.1-\frac{4 d A^{2}}{\left(A^{2}-d(B-1)\right)^{2}}\right\}} \int^{2}\left\{\sqrt{1-\left(\frac{A}{\left(\frac{A}{\sqrt{d}}\right)^{2}-(B-1)}\right)^{2}}\right\},\right.
\end{aligned}
$$

where we used that $(B-1) d-A^{2}>0$, see (11.21). Now, we assume that $d$ is sufficiently large, so we can use the Taylor expansion:

$$
\sqrt{1-\left(\frac{2\left(\frac{A}{\sqrt{d}}\right)}{\left(\frac{A}{\sqrt{d}}\right)^{2}-(B-1)}\right)^{2}} \approx 1-\frac{1}{2}\left(\frac{2\left(\frac{A}{\sqrt{d}}\right)}{\left(\frac{A}{\sqrt{d}}\right)^{2}-(B-1)}\right)^{2},
$$

then

$$
\kappa_{2} \approx \frac{-A^{2}}{\varepsilon d(B-1)} \text { and } \kappa_{1} \approx-\frac{(B-1)}{\varepsilon} .
$$

Now since $\kappa_{1}<\kappa_{2}$, i.e. $\frac{d(B-1)}{A^{2}}>\frac{1}{B-1} \Leftrightarrow(B-1)>\frac{A^{2}}{d(B-1)}$ and by (11.21), we have $B-1>$ $\max \left\{\frac{A^{2}}{d}, \frac{A^{2}}{d(B-1)}\right\}$. We pick

$$
\frac{A^{2}}{d}>\frac{A^{2}}{d(B-1)} \Leftrightarrow B>2 .
$$

Now (11.23) implies that $\kappa_{2}=\frac{-A^{2}}{\varepsilon d(B-1)}>\frac{-A^{2}}{\varepsilon d}$, and consequently, when verifying condition (T6) we can check the condition:

$$
\left\{\kappa \in \sigma(\boldsymbol{L} .) \backslash\{0\} ;-\frac{(B-1)}{\varepsilon}<\kappa<\frac{-A^{2}}{\varepsilon d}\right\} \neq \emptyset .
$$

Finally, under the hypothesis $B_{>} 2$, condition (11.21) is satisfied if $A^{2}<d$. In turn, since $A^{2}+2 \sqrt{d} A<3 d,(11.22)$ is satisfied if $B>4$.

11.2.1. Complete graphs. A complete graph $K_{n}$ is a connected graph on $n$ vertices where all vertices are of degree $n-1$. A complete graph has $\frac{n(n-1)}{2}$ edges. The eigenvalues of the 
Laplacian $\left[L_{J I}\right]_{J, I \in G_{N}^{0}}$ are $\mu_{I_{0}}=0, \mu_{I_{j}}=-n$ for $1 \leq j \leq n-1$, see e.g. [47, Section 5.1]. In the case $\boldsymbol{L}_{M}$ condition (11.24) becomes

$$
\frac{A^{2}}{d}<n \varepsilon<B-1
$$

This last condition is satisfied if $1<n \varepsilon<3$, since we have not imposed conditions on $\varepsilon$, we can pick $\varepsilon$ satisfying $\frac{1}{n}<\varepsilon<\frac{3}{n}$. In this case, there exist Turing patterns in $X_{N}$, $X_{M}$ with $M>N$ and in $X_{\infty}$.

In $X_{\infty}, \sigma(\boldsymbol{L}) \backslash\{0\}=\{-n\} \bigsqcup\{-n+1\}$. Then condition (11.24) becomes

$$
\frac{A^{2}}{d}<n \varepsilon<B-1 \text { and } \frac{A^{2}}{d}<(n-1) \varepsilon<B-1 \text {. }
$$

These two conditions cannot be satisfied simultaneously for some $\varepsilon>0$. We pick $\frac{3}{n}<\varepsilon<$ $\frac{3}{n-1}$, then there exists a Turing Pattern in $X_{\infty}$ but no in $X_{M}$ with $M \geq N$.

\section{REFERENCES}

[1] S. Albeverio, A. Yu. Khrennikov, V. M. Shelkovich, Theory of p-adic distributions: linear and nonlinear models. London Mathematical Society Lecture Note Series, 370. Cambridge University Press, Cambridge, 2010.

[2] B. Ambrosio, M. A. Aziz-Alaoui, V. L. E. Phan, Global attractor of complex networks of reactiondiffusion systems of Fitzhugh-Nagumo type, Discrete Contin. Dyn. Syst., Ser. B 23 (2018), No. 9, 3787-3797.

[3] Alexandra V. Antoniouk, Klaudia Oleschko, Anatoly N. Kochubei, Andrei Yu Khrennikov, A stochastic p-adic model of the capillary flow in porous random medium, Phys. A 505 (2018), 763-777.

[4] Alexandra V. Antoniouk, Andrei Yu. Khrennikov, Anatoly N. Kochubei, Multidimensional nonlinear pseudo-differential evolution equation with p-adic spatial variables, J. Pseudo-Differ. Oper. Appl. 11 (2020), no. 1, 311-343.

[5] V. A. Avetisov, A. Kh. Bikulov, V. A. Osipov, p-adic description of characteristic relaxation in complex systems, J. Phys. A 36 (2003), no. 15, 4239-4246.

[6] V. A. Avetisov, A. H. Bikulov, S. V. Kozyrev, V. A. Osipov, p-adic models of ultrametric diffusion constrained by hierarchical energy landscapes, J. Phys. A 35 (2002), no. 2, 177-189.

[7] O. M. Becker, M. Karplus, The topology of multidimensional protein energy surfaces: theory and application to peptide structure and kinetics, J. Chem.Phys. 106 (1997), 1495-1517.

[8] A. D. Bendikov, A. A. Grigor'yan, K. Pittè, V. Vëss, Isotropic Markov semigroups on ultra-metric spaces, Russian Math. Surveys 69 (2014), no. 4, 589-680.

[9] Alexander Bendikov, Heat kernels for isotropic-like Markov generators on ultrametric spaces: a survey, p-Adic Numbers Ultrametric Anal. Appl. 10 (2018), no. 1, 1-11.

[10] A. Bendikov, P. Krupski, On the spectrum of the hierarchical Laplacian, Potential Analysis 41 (2014), no. $4,1247-1266$.

[11] Gregory Berkolaiko, Kuchment Peter, Introduction to quantum graphs, American Mathematical Society, Providence, RI, 2013.

[12] R. M. Blumenthal, R. K. Getoor, Markov processes and potential Theory, Academic Press, New York and London, 1968.

[13] S. Boccaletti, V. Latora, Y. Moreno, M. Chavez, D.-U. Hwang, Complex networks: structure and dynamics, Phys. Rep. 424 (2006), no. 4-5, 175-308. 
[14] Soon-Yeong Chung, Jae-Hwang Lee, Blow-up for discrete reaction-diffusion equations on networks, Appl. Anal. Discrete Math. 9 (2015), No. 1, 103-119.

[15] Stewart N. Ethier, Thomas G. Kurtz, Markov Processes - Characterization and convergence, Wiley Series in Probability and Mathematical Statistics, John Wiley \& Sons, New York, 1986.

[16] H. Frauenfelder, S. S. Chan, W. S. Chan (eds), The Physics of Proteins, Springer-Verlag, 2010.

[17] Paul R. Halmos, Measure Theory, D. Van Nostrand Company, 1950.

[18] Yusuke Ide, Hirofumi Izuhara, Takuya Machida, Turing instability in reaction-diffusion models on complex networks, Physica A 457 (2016), 331-347.

[19] Andrei Khrennikov, Klaudia Oleschko, Maria de Jesús Correa López, Application of p-adic wavelets to model reaction-diffusion dynamics in random porous media, J. Fourier Anal. Appl. 22 (2016), no. 4, 809-822.

[20] Andrei Khrennikov, Klaudia Oleschko, María de Jesús Correa López, Modeling fluid's dynamics with master equations in ultrametric spaces representing the treelike structure of capillary networks, Entropy 18 (2016), no. 7, Paper No. 249, 28 pp.

[21] Andrei Yu. Khrennikov, Anatoly N. Kochubei, $p$-adic analogue of the porous medium equation, $J$. Fourier Anal. Appl. 24 (2018), No. 5, 1401-1424.

[22] Andrei Khrennikov, Sergei Kozyrev, W. A. Zúñiga-Galindo, Ultrametric Equations and its Applications, Encyclopedia of Mathematics and its Applications (168), Cambridge University Press, 2018.

[23] Neal Koblitz, p-adic Numbers, p-adic Analysis, and Zeta-Functions, Graduate Texts in Mathematics No. 58, Springer-Verlag, 1984.

[24] Anatoly N. Kochubei, Pseudo-differential equations and stochastics over non-Archimedean fields, Marcel Dekker, Inc., New York, 2001.

[25] S. V. Kozyrev, Wavelets and spectral analysis of ultrametric pseudodifferential operators, Mat. Sb.198 (2007), no. 1, 97-116.

[26] S. V. Kozyrev, Methods and Applications of Ultrametric and p-Adic Analysis: From Wavelet Theory to Biophysics, Proceedings of the Steklov Institute of Mathematics, 274 (2011), 1-84.

[27] I. Lengyel, I. R. Epstein, Modeling of Turing structure in the chlorite-iodide-malonic acid-starch reaction system, Science 251 (1991), 650-652.

[28] Milan Miklavčič, Applied functional analysis and partial differential equations, World Scientific Publishing Co., Inc., River Edge, NJ, 1998.

[29] M. Mocarlo Zheng, Bin Shao, Qi Ouyang, Identifying network topologies that can generate Turing pattern, J. Theor. Biol. 408 (2016), 88-96 .

[30] Delio Mugnolo, Semigroup methods for evolution equations on networks. Understanding Complex Systems, Springer, Cham, 2014.

[31] J. D. Murray, Mathematical biology. II. Spatial models and biomedical applications. Third edition. Springer-Verlag, New York, 2003.

[32] Nakao Hiroya and Mikhailov Alexander S., Turing patterns in network-organized activator-inhibitor systems, Nature Physics 6 (2010), 544-550.

[33] G. Nicolis, I. Prigogine, Self-organization in Non-equilibrium Systems. Wiley Interscience, New-York (1977).

[34] K. Oleschko, Andrei Khrennikov, Transport through a network of capillaries from ultrametric diffusion equation with quadratic nonlinearity, Russ. J. Math. Phys. 24 (2017), No. 4, 505-516.

[35] K. Oleschko, A. Yu. Khrennikov, Applications of $p$-adics to geophysics: linear and quasilinear diffusion of water-in-oil and oil-in-water emulsions, Theor. Math. Phys. 190 (2017), No. 1, 154-163.

[36] H. G. Othmer, L. E. Scriven, Instability and dynamic pattern in cellular networks, J. Theor. Biol. 32 (1971), 507-537. 
[37] H. G. Othmer, L. E. Scriven, Nonlinear aspects of dynamic pattern in cellular networks, J. Theor. Biol. 43 (1974), 83-112.

[38] A. Pazy, Semigroups of linear operators and applications to partial differential equations. Applied Mathematical Sciences, 44. Springer-Verlag, New York, 1983.

[39] Benoît Perthame, Parabolic equations in biology. Growth, reaction, movement and diffusion. Lecture Notes on Mathematical Modelling in the Life Sciences. Springer, Cham, 2015.

[40] I. Prigogine, R. Lefever, Symmetry breaking instabilities in dissipative systems II., J. Chem. Phys. 48 (1968), 1695-1700.

[41] Angela Slavova, Pietro Zecca, Complex behavior of polynomial FitzHugh-Nagumo cellular neural network model, Nonlinear Anal., Real World Appl. 8 (2007), No. 4, 1331-1340.

[42] M. H. Taibleson, Fourier analysis on local fields, Princeton University Press, 1975.

[43] Kazuaki Taira, Boundary value problems and Markov processes. Second edition. Lecture Notes in Mathematics, 1499. Springer-Verlag, 2009.

[44] Anselmo Torresblanca-Badillo, W. A. Zúñiga-Galindo, Ultrametric diffusion, exponential landscapes, and the first passage time problem, Acta Appl. Math. 157 (2018), 93-116.

[45] Anselmo Torresblanca-Badillo, W. A. Zúñiga-Galindo, Non-Archimedean pseudodifferential operators and Feller semigroups, p-Adic Numbers Ultrametric Anal. Appl. 10 (2018), no. 1, 57-73.

[46] A. M. Turing, The chemical basis of morphogenesis, Phil. Trans. R. Soc. Lond. B 237 (1952), 37-72.

[47] Piet Van Mieghem, Graph spectra for complex networks, Cambridge University Press, Cambridge, 2011.

[48] V. S. Vladimirov, I. V. Volovich, E. I. Zelenov, p-adic analysis and mathematical physics, World Scientific, 1994.

[49] Joachim von Below, José A. Lubary, Instability of stationary solutions of reaction-diffusion-equations on graphs, Result. Math. 68 (2015), No. 1-2, 171-201.

[50] Hongyong Zhao, Xuanxuan Huang, Xuebing Zhang, Turing instability and pattern formation of neural networks with reaction-diffusion terms, Nonlinear Dyn. 76 (2014), No. 1, 115-124.

[51] W. A. Zúñiga-Galindo, Pseudodifferential equations over non-Archimedean spaces. Lectures Notes in Mathematics 2174, Springer, Cham, 2016.

[52] W. A. Zúñiga-Galindo, Non-archimedean replicator dynamics and Eigen's paradox, J. Phys. A 51 (2018), no. 50, 505601, 26 pp.

[53] W. A. Zúñiga-Galindo, Non-Archimedean Reaction-Ultradiffusion Equations and Complex Hierarchic Systems, Nonlinearity 31 (2018), no. 6, 2590-2616.

[54] W. A. Zúñiga-Galindo, Eigen's Paradox and the Quasispecies Model in a Non-Archimedean Framework. arXiv:2111.01745.

Centro de Investigación y de Estudios Avanzados del Instituto Politécnico Nacional, Departamento de Matemáticas, Unidad Querétaro, Libramiento Norponiente \#2000, Fracc. Real de Juriquilla. Santiago de Querétaro, Qro. 76230, México.

Email address: wazuniga@math.cinvestav.edu.mx 